\title{
Review \\ Maternal Reproductive Toxicity of Some Essential Oils and Their Constituents
}

\author{
Noura S. Dosoky ${ }^{1, *(\mathbb{D})}$ and William N. Setzer ${ }^{1,2}$ (D) \\ 1 Aromatic Plant Research Center, Lehi, UT 84043, USA \\ 2 Department of Chemistry, University of Alabama in Huntsville, Huntsville, AL 35899, USA; \\ wsetzer@chemistry.uah.edu \\ * Correspondence: ndosoky@aromaticplant.org; Tel.: +1-256-457-0135
}

Citation: Dosoky, N.S.; Setzer, W.N. Maternal Reproductive Toxicity of Some Essential Oils and Their Constituents. Int. J. Mol. Sci. 2021, 22, 2380. https://doi.org/10.3390/ ijms22052380

Academic Editor:

Marie-Laure Fauconnier

Received: 2 February 2021

Accepted: 24 February 2021

Published: 27 February 2021

Publisher's Note: MDPI stays neutral with regard to jurisdictional claims in published maps and institutional affiliations.

Copyright: (c) 2021 by the authors. Licensee MDPI, Basel, Switzerland. This article is an open access article distributed under the terms and conditions of the Creative Commons Attribution (CC BY) license (https:// creativecommons.org/licenses/by/ $4.0 /)$.

\begin{abstract}
Even though several plants can improve the female reproductive function, the use of herbs, herbal preparations, or essential oils during pregnancy is questionable. This review is focused on the effects of some essential oils and their constituents on the female reproductive system during pregnancy and on the development of the fetus. The major concerns include causing abortion, reproductive hormone modulation, maternal toxicity, teratogenicity, and embryo-fetotoxicity. This work summarizes the important studies on the reproductive effects of essential oil constituents anethole, apiole, citral, camphor, thymoquinone, trans-sabinyl acetate, methyl salicylate, thujone, pulegone, $\beta$-elemene, $\beta$-eudesmol, and costus lactone, among others.
\end{abstract}

Keywords: essential oils; pregnancy; anethole; trans-sabinyl acetate; camphor; methyl salicylate; thujone; pulegone; citral; apiole

\section{Introduction}

The female reproductive cycle involves a very complex sequence of changes in the uterus, ovaries, breasts, and regulatory hormone levels. Several mechanisms, metabolic pathways, and enzymes are involved in controlling and regulating reproductive hormone levels in the blood. During the reproductive cycle, these endogenous hormones are responsible for preparing for implantation and for milk production [1,2]. Out of concern of adversely affecting the unborn child, some pregnant women prefer to use herbs, herbal preparations, or oils rather than conventional medication to treat pregnancy-related symptoms (morning sickness, nausea, vomiting, heartburn, etc.) $[3,4]$. Indeed, several plants can improve the female reproductive function and some are beneficial during pregnancy, childbirth, and postpartum [5,6]. Similarly, essential oils (EOs) are generally safe, and many oils have a generally recognized as safe (GRAS) status. However, the use of herbs and EOs during pregnancy is a highly controversial matter. It is worth mentioning that it is the individual composition of an EO and the possible hazardousness of a single or a group of constituents that determine their medical and therapeutic usage. Some EO-containing plant species are highly variable and may produce several EO chemotypes with different EO compositions of which one or some chemotypes possess potential maternal reproductive toxicity. Causing abortion is a major concern. It is generally believed that EOs extracted from emmenagogic plants are dangerous or unsafe in pregnancy, as they might cause menstrual bleeding and lead to miscarriage. Yet, that is not always true. The oils do not necessarily carry the same activity as the whole plant. Regardless of their ability to promote menstruation, there is no decisive evidence that these oils are abortifacient in aromatherapy amounts. For instance, the whole plants of savin, pennyroyal, tansy, and rue can induce miscarriage and their oils were on the list of abortifacient oils at some point [7]. Thus far, these oils showed no activity on uterine muscle of isolated human uterus [8] and did not cause fetal death $[9,10]$. Still, these facts do not prove the safety of these oils. Since the 
toxicity of many plants and EOs has not been studied yet, the concern cannot be completely dismissed.

Another major concern about essential oils and their constituents is mimicking, interfering with, or antagonizing the action of reproductive hormones, which in turn could disrupt the reproductive and developmental processes [11]. Some EO constituents could affect the outcome of pregnancy through causing maternal toxicity, teratogenicity, embryofetotoxicity, or anti-angiogenicity [12]. Since angiogenesis is essential for a successful pregnancy, anti-angiogenic oil constituents carry the risk of causing preeclampsia, growth restriction, and fetal death [13,14]. Moreover, at high doses, teratogenic oils or components can cause birth defects of a structural nature that arise during embryonic development [15]. Generally, EO components can cross the placenta to the fetal circulation due to their low molecular weight, protein binding ability, and lipophilicity and can cause fetotoxicity [16]. Components that cross the placenta are more likely to affect the fetal central nervous system (CNS) since it is underdeveloped [17]. Unsaturated compounds like cinnamaldehyde, citral, and $\beta$-pulegone act via interacting with the lipids in embryo cell membranes [18]. Subcutaneous injection of 1,8-cineole (at $500 \mathrm{mg} / \mathrm{kg}$ for four days) to pregnant rats affected the fetal liver enzymes activity [19]. Similar to crossing the placenta, most flavor and EO constituents are expected to pass into breast milk via passive diffusion [20]. In lactating mice, constituents of sandalwood oil were able to pass to maternal milk and affected infant hepatic metabolic enzymes [21].

This review has been prepared based on a comprehensive survey of major scientific databases for the effects of some EOs and their constituents on the female reproductive system during pregnancy and on the development of the fetus. Figure 1 presents the chemical structures of key essential oil components discussed in this review, while the chemical composition of these oils is summarized in Table 1. It should be noted that because of the ethical and safety issues in human toxicity testing, a substantial weight is usually given to animal testing for reproductive toxicity despite the major differences in reproductive physiologies. EOs or components that showed animal reproductive toxicity should either be prohibited (strictly avoided) or restricted (used with some degree of caution) during pregnancy and lactation.

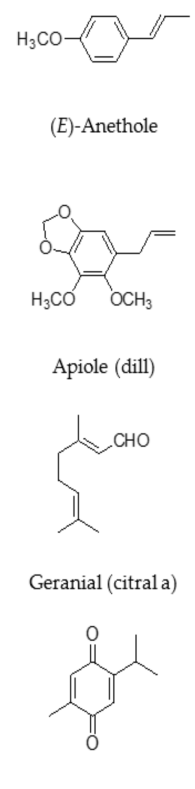

Thymoquinone
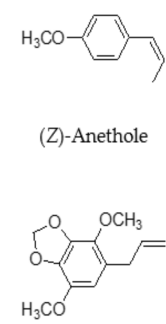

Apiole (parsley)

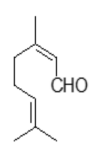

Neral (citralb)

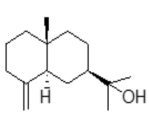

$\beta$-Eudesmol

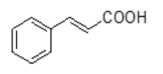

(2E)-Cinnamic acid
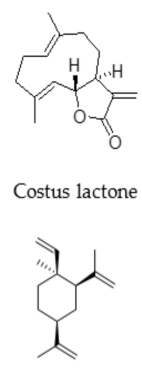

$\beta$-Elemene

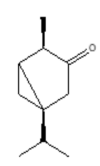

$\alpha$-Thujone

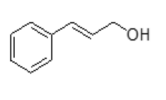

Cinnamyl alcohol
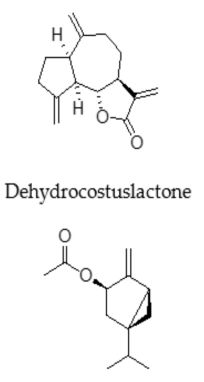

Sabinyl acetate

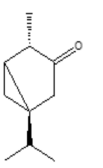

$\beta$-Thujone

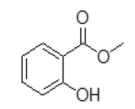

Methyl salicylate

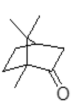

Camphor

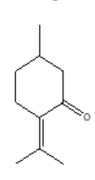

Pulegone

Figure 1. Chemical structures of key essential oil components discussed in this review. 
Table 1. Potentially toxic essential oils and their toxic constituents discussed in this review.

\begin{tabular}{|c|c|c|c|c|c|c|c|c|}
\hline EO & Botanical Name & Family & Part Used & Hazard(s) & $\begin{array}{c}\text { Toxic } \\
\text { Component(s) }\end{array}$ & Oil Composition & $\begin{array}{l}\text { Maximum Oral } \\
\text { Dose in } \\
\text { Pregnancy [22] }\end{array}$ & Ref. \\
\hline Anise or aniseed & Pimpinella anisum L. & Apiaceae & Seeds & $\begin{array}{l}\text { Reproductive } \\
\text { hormone } \\
\text { modulation }\end{array}$ & (E)-Anethole & $\begin{array}{c}(E) \text {-anethole }(75.2-96.1 \%), \\
d \text {-limonene }(\operatorname{tr}-4.9 \%), \text { and } \\
\text { estragole }(0.5-5.0 \%)\end{array}$ & - & {$[23,24]$} \\
\hline Aniseed Myrtle & $\begin{array}{c}\text { Syzygium anisatum } \\
\text { (Vickery) Craven and } \\
\text { Biffin }\end{array}$ & Myrtaceae & Leaves & $\begin{array}{l}\text { Reproductive } \\
\text { hormone } \\
\text { modulation }\end{array}$ & (E)-Anethole & $\begin{array}{c}(E) \text {-anethole }(95.0 \%), \text { and } \\
\text { estragole }(4.4 \%)\end{array}$ & - & [25] \\
\hline Araucaria & $\begin{array}{l}\text { Neocallitropsis pancheri } \\
\text { (Carriere) de Laub. } \\
\text { (synonym: Callitropsis } \\
\text { araucarioides Compton, } \\
\text { and Neocallitropsis } \\
\text { araucarioides (Compton) } \\
\text { Florin) }\end{array}$ & Cupressaceae & Wood & $\begin{array}{c}\text { Fetotoxic, } \\
\text { anti-angiogenic }\end{array}$ & $\beta$-Eudesmol & $\begin{array}{c}\beta \text {-eudesmol (25.9\%), } \gamma \text {-eudesmol } \\
(19.0 \%), \alpha \text {-eudesmol }(13.3 \%) \\
\text { guaiol }(6.0 \%), \text { elemol }(5.0 \%), \text { and } \\
\beta \text {-bisabolenol }(4.9 \%)\end{array}$ & - & [26] \\
\hline $\begin{array}{l}\text { Atractylis } \\
\text { (Cang-zhu } \\
\text { atractylodes) }\end{array}$ & $\begin{array}{l}\text { Atractylodes lancea } \\
\text { (Thunb.) DC }\end{array}$ & Asteraceae & Roots & $\begin{array}{l}\text { Anti-angiogenic, } \\
\text { fetotoxic }\end{array}$ & $\begin{array}{c}\beta \text {-Elemene and } \\
\beta \text {-eudesmol }\end{array}$ & $\begin{array}{c}\beta \text {-eudesmol }(26.0 \%), \beta \text {-elemene } \\
(18.0 \%), \text { hinesol }(10.0 \%), \text { and } \\
\text { elemol }(6.0 \%)\end{array}$ & - & [22] \\
\hline $\begin{array}{l}\text { Australian Lemon } \\
\text { balm } \\
\text { (lemon-scented } \\
\text { ironbark) }\end{array}$ & $\begin{array}{l}\text { Eucalyptus staigeriana F. v. } \\
\text { Muell. ex F. M. Bailey }\end{array}$ & Myrtaceae & Leaves & Teratogenicity & Citral & $\begin{array}{l}d \text {-limonene+ } \beta \text {-phellandrene } \\
(30.5 \%), \text { geranial }(9.9 \%), \text { neral } \\
(7.7 \%), \alpha \text {-phellandrene }(7.1 \%) \\
\text { and terpinolene }(6.6 \%)\end{array}$ & $\begin{array}{c}238 \mathrm{mg} / \text { day based } \\
\text { on } 17.6 \% \text { citral } \\
\text { content }\end{array}$ & [22] \\
\hline $\begin{array}{l}\text { Basil oil (estragole } \\
\text { chemotype) }\end{array}$ & Ocimum basilicum $\mathrm{L}$. & Lamiaceae & Leaves & $\begin{array}{l}\text { Potentially } \\
\text { carcinogenic }\end{array}$ & $\begin{array}{l}\text { Estragole and } \\
\text { methyleugenol }\end{array}$ & $\begin{array}{c}\text { estragole }(73.4-87.4 \%) \text {, linalool } \\
(\operatorname{tr}-8.6 \%), \text { and } 1,8 \text {-cineole } \\
(0.6-6.0 \%)\end{array}$ & - & [23] \\
\hline Bitter Fennel & $\begin{array}{l}\text { Foeniculum vulgare Mill. } \\
\text { subsp. capillaceum Gilib. }\end{array}$ & Apiaceae & Seeds & $\begin{array}{l}\text { Reproductive } \\
\text { hormone } \\
\text { modulation }\end{array}$ & $(E)$-anethole & $\begin{array}{c}(E) \text {-anethole }(52.5-84.3 \%) \\
\text { fenchone }(4.0-24.0 \%), \alpha \text {-pinene } \\
\text { (tr-10.4\%), } d \text {-limonene }(0.5-9.4 \%) \\
\text { and estragole }(2.8-6.5 \%)\end{array}$ & - & [23] \\
\hline $\begin{array}{l}\text { Black seed (black } \\
\text { cumin or black } \\
\text { caraway) }\end{array}$ & Nigella sativa L. & Ranunculaceae & Seeds & Fetotoxic & Thymoquinone & $\begin{array}{c}\text { thymoquinone }(26.8-54.8 \%) \\
p \text {-cymene }(14.7-38.0 \%), \\
\text { longifolene }(1.2-10.2 \%), \text { and } \\
\alpha \text {-thujene }(1.3-10.1 \%) \text { as the main } \\
\text { constituents. }\end{array}$ & - & [27] \\
\hline
\end{tabular}


Table 1. Cont.

\begin{tabular}{|c|c|c|c|c|c|c|c|c|}
\hline EO & Botanical Name & Family & Part Used & Hazard(s) & $\begin{array}{c}\text { Toxic } \\
\text { Component(s) }\end{array}$ & Oil Composition & $\begin{array}{l}\text { Maximum Oral } \\
\text { Dose in } \\
\text { Pregnancy [22] }\end{array}$ & Ref. \\
\hline $\begin{array}{c}\text { Blue Cypress } \\
\text { (Northern cypress } \\
\text { pine) }\end{array}$ & $\begin{array}{c}\text { Callitris intratropica R.T. } \\
\text { Baker and H.B. Sm. }\end{array}$ & Cupressaceae & Wood & $\begin{array}{c}\text { Fetotoxic, } \\
\text { anti-angiogenic }\end{array}$ & $\beta$-Eudesmol & $\begin{array}{c}\beta \text {-eudesmol }(14.4 \%), \\
\text { dihydrocolumellarin }(14.0 \%), \\
\text { guaiol }(13.7 \%), \gamma \text {-eudesmol } \\
(9.1 \%), \alpha \text {-eudesmol }(7.6 \%), \\
\text { guaiazulene }(6.2 \%), \text { chamazulene } \\
(5.6 \%)\end{array}$ & - & [28] \\
\hline $\begin{array}{l}\text { Buchu (diosphenol } \\
\text { chemotype) }\end{array}$ & $\begin{array}{c}\text { Agathosma betulina } \\
\text { Bergius }\end{array}$ & Rutaceae & Leaves & $\begin{array}{l}\text { Abortifacient; } \\
\text { hepatotoxicity }\end{array}$ & Pulegone & $\begin{array}{c}\text { isomenthone }(4.6-29.1 \%), \\
\text { limonene }(11.6-28.2 \%), \\
\text { disophenol }(12.0-26.3 \%), \\
\text { menthone }(2.5-25.0 \%), \\
\text { c-diosphenol }(10.3-23.3 \%), \text { and } \\
\text { 8-mercapto- } p \text {-menthan-3-one(cis- } \\
\text { trans) } \\
(0.7-6.6 \%)\end{array}$ & - & [29] \\
\hline $\begin{array}{l}\text { Buchu (pulegone } \\
\text { chemotype) }\end{array}$ & Agathosma crenulata $\mathrm{L}$. & Rutaceae & Leaves & abortifacient & Pulegone & $\begin{array}{c}\text { (1R)-(+)- } \beta \text {-pulegone }(31.6-73.2 \%), \\
\text { isomenthone }(3.6-27.6 \%) \\
\text { limonene }(2.1-17.2 \%) \\
(E)-8 \text {-acetylthio- } p \text {-menthan-3-one } \\
(0.4-10.4 \%), \text { and menthone } \\
(1.3-7.0 \%)\end{array}$ & - & {$[29,30]$} \\
\hline Carrot seed & $\begin{array}{l}\text { Daucus carota L. subsp. } \\
\text { sativus Hoffm. }\end{array}$ & Apiaceae & Seeds & $\begin{array}{l}\text { antigestational } \\
\text { effects }\end{array}$ & & $\begin{array}{c}\text { carotol }(36.1-73.1 \%), \alpha \text {-pinene } \\
(0.9-11.2 \%), \text { dauca-4,8-diene } \\
(1.6-5.9 \%), \text { and } \beta \text {-caryophyllene } \\
(0.7-5.6 \%)\end{array}$ & - & [31] \\
\hline $\begin{array}{l}\text { Cassia (Chinese or } \\
\text { false cinnamon) }\end{array}$ & $\begin{array}{l}\text { Cinnamomum cassia }(\mathrm{L} .) \mathrm{J} . \\
\text { Presl (synonym: } \\
\text { Cinnamomum aromaticum } \\
\text { Nees) }\end{array}$ & Lauraceae & $\begin{array}{l}\text { Leaves, } \\
\text { terminal } \\
\text { branches } \\
\text { and bark }\end{array}$ & $\begin{array}{l}\text { Embryotoxicity, } \\
\text { reproductive } \\
\text { toxicity }\end{array}$ & $\begin{array}{l}\text { Methyleugenol } \\
\text { and } \\
\text { cinnamaldehyde }\end{array}$ & $\begin{array}{c}\text { (E)-cinnamaldehyde }(73.2-89.4 \%), \\
\text { (Z)-cinnamaldehyde }(0.8-12.3 \%), \\
\text { and }(E) \text {-cinnamyl acetate } \\
(0.1-5.4 \%) \text { while in the leaf oil } \\
(E) \text {-cinnamaldehyde }(54.6-90.1 \%), \\
\text { (E)-cinnamyl acetate }(1.4-12.5 \%) \\
\text { (Z)-cinnamaldehyde }(0.4-10.5 \%) \\
\text { and benzaldehyde }(1.1-6.3 \%)\end{array}$ & - & [32] \\
\hline
\end{tabular}


Table 1. Cont.

\begin{tabular}{|c|c|c|c|c|c|c|c|c|}
\hline EO & Botanical Name & Family & Part Used & Hazard(s) & $\begin{array}{c}\text { Toxic } \\
\text { Component(s) }\end{array}$ & Oil Composition & $\begin{array}{l}\text { Maximum Oral } \\
\text { Dose in } \\
\text { Pregnancy [22] }\end{array}$ & Ref. \\
\hline $\begin{array}{c}\text { Chaste tree } \\
\text { (Monk's pepper) }\end{array}$ & Vitex agnus-castus L. & Verbenaceae & Leaves & $\begin{array}{l}\text { Reproductive } \\
\text { hormone } \\
\text { modulation }\end{array}$ & $\begin{array}{l}\text { The oil may } \\
\text { contain } \\
\text { methyleugenol }\end{array}$ & $\begin{array}{c}\text { Leaf EO: } 1,8 \text {-cineole }(15.6-35.2 \%), \\
\text { sabinene }(6.9-17.1 \%), \alpha \text {-pinene } \\
(1.0-13.9 \%), \alpha \text {-terpineol } \\
(1.4-9.2 \%), \gamma \text {-elemene }(0-9.1 \%) \text {, } \\
\beta \text {-selinene }(0-9.0 \%), \\
\beta \text {-caryophyllene }(2.3-8.9 \%), \\
(Z)-\beta \text {-farnesene }(0-8.6 \%), \\
\text { citronellyl acetate }(0.3-7.8 \%), \text { and } \\
\text { citronellic acid }(0-6.6 \%) \\
\text { Seed EO: sabinene }(7.1-44.1 \%), \\
1,8 \text {-cineole }(8.4-23.3 \%), \alpha \text {-pinene } \\
(1.2-23.1 \%), \gamma \text {-elemene }(0-17.0 \%), \\
(E)-\beta \text {-farnesene }(0-10.3 \%), \\
\beta \text {-caryophyllene }(0.8-9.3 \%), \\
\alpha \text {-terpineol }(0.2-9.3 \%), \text { limonene } \\
(0.5-7.4 \%),(Z)-\beta \text {-farnesene } \\
(0-6.9 \%), \text { citronellyl acetate } \\
(0.2-6.0 \%), \beta \text {-selinene }(0-6.0 \%), \\
\text { and } \beta \text {-myrcene }(0-5.6 \%) .\end{array}$ & - & {$[33,34]$} \\
\hline Cinnamon bark & $\begin{array}{l}\text { Cinnamomum verum J. } \\
\text { Presl. (Synonym: } \\
\text { Cinnamomum zeylanicum } \\
\text { Blume) }\end{array}$ & Lauraceae & $\begin{array}{l}\text { Dried } \\
\text { inner bark } \\
\text { of young } \\
\text { trees }\end{array}$ & Embryotoxicity & $\begin{array}{c}(E)- \\
\text { Cinnamaldehyde }\end{array}$ & $\begin{array}{c}(E) \text {-cinnamaldehyde }(63.1-75.7 \%), \\
\text { eugenol }(2.0-13.3 \%), \\
(E) \text {-cinnamyl acetate }(0.3-10.6 \%), \\
\text { linalool }(0.2-7.0 \%), \text { and } \\
\beta \text {-caryophyllene }(1.3-5.8 \%)\end{array}$ & - & {$[23,24]$} \\
\hline Costus & $\begin{array}{l}\text { Saussurea costus (Falc.) } \\
\text { Lipsch. (synonym: } \\
\text { Aplotaxis lappa Decne., } \\
\text { Aucklandia costus Falc., } \\
\text { Saussurea lappa (Decne) } \\
\text { C.B. Clarke) }\end{array}$ & Asteraceae & Dried roots & $\begin{array}{c}\text { Fetotoxicity, } \\
\text { anti-angiogenicity }\end{array}$ & $\begin{array}{l}\text { Costunolide and } \\
\text { dehydrocostus } \\
\text { lactone }\end{array}$ & $\begin{array}{c}\text { aplotaxene }(20.0 \%), \\
\text { dihydrocostus lactone }(15.0 \%), \\
\text { costusic acid }(14.0 \%) \text {, costunolide } \\
(11.0 \%) \text {, dehydrocostus lactone } \\
(6.0 \%) \text {, and } \\
\text { dihydrodehydrocostus lactone } \\
(6.0 \%)\end{array}$ & - & {$[35]$} \\
\hline
\end{tabular}


Table 1. Cont.

\begin{tabular}{|c|c|c|c|c|c|c|c|c|}
\hline EO & Botanical Name & Family & Part Used & Hazard(s) & $\begin{array}{c}\text { Toxic } \\
\text { Component(s) }\end{array}$ & Oil Composition & $\begin{array}{l}\text { Maximum Oral } \\
\text { Dose in } \\
\text { Pregnancy [22] }\end{array}$ & Ref. \\
\hline Dalmatian Sage & Salvia officinalis L. & Lamiaceae & Leaves & embryotoxic & $\begin{array}{l}\text { Camphor, } \\
\text { thujones }\end{array}$ & $\begin{array}{c}\text { camphor }(7.3-50.2 \%), \alpha \text {-thujone } \\
(13.1-48.5 \%), \text { borneol }(1.5-23.9 \%), \\
\text { 1,8-cineole }(1.8-21.7 \%), \beta \text {-thujone } \\
(3.9-19.1 \%), \beta \text {-caryophyllene } \\
(0.2-9.7 \%), \text { camphene }(0-8.6 \%), \\
\alpha \text {-pinene }(0-8.0 \%) \text { and bornyl } \\
\text { acetate }(0.3-5.7 \%)\end{array}$ & - & [36] \\
\hline $\begin{array}{l}\text { Feverfew } \\
\text { (nosebleed or } \\
\text { midsummer } \\
\text { daisy) }\end{array}$ & $\begin{array}{l}\text { Tanacetum parthenium (L.) } \\
\text { Sch. Bip. (synonym: } \\
\text { Chrysanthemum } \\
\text { parthenium (L.) Bernh.) }\end{array}$ & Asteraceae & Leaves & $\begin{array}{l}\text { Unsafe, } \\
\text { moderately } \\
\text { neurotoxic }\end{array}$ & Camphor & $\begin{array}{c}\text { camphor }(28.0-44.2 \%), \\
(E) \text {-chrysanthenyl acetate } \\
(22.9-30.2 \%), \text { camphene } \\
(5.4-7.7 \%) \text {, and germacrene D } \\
(0.7-4.6 \%)\end{array}$ & - & [37] \\
\hline Genipi (Genepi) & $\begin{array}{l}\text { Artemisia genepi Weber. } \\
\text { (synonym: A. spicata } \\
\text { Wulfen, and A. mutellina } \\
\text { Vill.) }\end{array}$ & Asteraceae & $\begin{array}{l}\text { Aerial } \\
\text { parts }\end{array}$ & Neurotoxic & Thujone & $\begin{array}{c}\alpha \text {-thujone }(79.8 \%) \text { and } \beta \text {-thujone } \\
(10.4 \%)\end{array}$ & - & [38] \\
\hline Great Mugwort & Artemisia arborescens L. & Asteraceae & $\begin{array}{c}\text { Aerial } \\
\text { parts }\end{array}$ & Neurotoxic & Thujone & $\begin{array}{c}\beta \text {-thujone (34.0\%), chamazulene } \\
(22.4 \%), \text { and camphor }(11.8 \%)\end{array}$ & - & [39] \\
\hline $\begin{array}{c}\text { Green Yarrow } \\
\text { (Ligurian yarrow) }\end{array}$ & $\begin{array}{c}\text { Achillea nobilis L. } \\
\text { (synonym: A. ligustica Vis. } \\
\text { ex Nym.) }\end{array}$ & Asteraceae & $\begin{array}{l}\text { Aerial } \\
\text { parts of the } \\
\text { flowering } \\
\text { plant }\end{array}$ & Abortifacient & $\begin{array}{l}\text { Sabinyl acetate; } \\
\text { camphor }\end{array}$ & $\begin{array}{c}\text { camphor }(13.7 \%) \text { artemisia } \\
\text { alcohol }(9.2 \%) \text {, germacrene } \mathrm{D} \\
(8.8 \%) \text {, artemisia ketone }(8.7 \%) \\
\text { and viridiflorol }(5.7 \%)\end{array}$ & - & {$[22]$} \\
\hline $\begin{array}{l}\text { Ho leaf (camphor } \\
\text { chemotype) }\end{array}$ & $\begin{array}{l}\text { Cinnamomum camphora } \\
\text { (L.) J.Presl }\end{array}$ & Lauraceae & Leaves & Neurotoxic & Camphor & $\begin{array}{c}\text { camphor }(37.8-84.1 \%), 1,8 \text {-cineole } \\
(1.0-12.0 \%) \text {, and terpinen-4-ol } \\
(0.9-6.3 \%)\end{array}$ & - & {$[40]$} \\
\hline $\begin{array}{l}\text { Honey Myrtle } \\
\text { (Marsh honey } \\
\text { myrtle) }\end{array}$ & Melaleuca teretifolia Endl. & Myrtaceae & Leaves & Teratogenicity & Citral & $\begin{array}{c}\text { geranial }(37.5 \%) \text {, neral }(29.0 \%) \\
\text { and } \beta \text {-myrcene }(10.9 \%)\end{array}$ & $\begin{array}{l}63 \mathrm{mg} / \text { day based } \\
\text { on } 66.5 \% \text { citral } \\
\text { content }\end{array}$ & {$[22]$} \\
\hline $\begin{array}{c}\text { Hyssop } \\
\text { (pinocamphone } \\
\text { chemotype) }\end{array}$ & Hyssopus officinalis L. & Lamiaceae & $\begin{array}{l}\text { Leaves and } \\
\text { flowering } \\
\text { tops }\end{array}$ & $\begin{array}{l}\text { Neurotoxicity; } \\
\text { carcinogen }\end{array}$ & $\begin{array}{l}\text { Pinocamphone, } \\
\text { methyleugenol }\end{array}$ & $\begin{array}{l}\text { pinocamphone }(31.2-42.7 \%) \\
\text { isopinocamphone }(30.9-39.2 \%) \\
\text { and } \beta \text {-pinene }(4.0-8.8 \%)\end{array}$ & - & {$[37,41]$} \\
\hline
\end{tabular}


Table 1. Cont.

\begin{tabular}{|c|c|c|c|c|c|c|c|c|}
\hline EO & Botanical Name & Family & Part Used & Hazard(s) & $\begin{array}{c}\text { Toxic } \\
\text { Component(s) }\end{array}$ & Oil Composition & $\begin{array}{l}\text { Maximum Oral } \\
\text { Dose in } \\
\text { Pregnancy [22] }\end{array}$ & Ref. \\
\hline $\begin{array}{l}\text { Indian dill seed } \\
\quad \text { (Sowa) }\end{array}$ & $\begin{array}{l}\text { Anethum sowa Roxb. ex } \\
\text { Flem. }\end{array}$ & Apiaceae & Seeds & $\begin{array}{l}\text { hepatotoxic, } \\
\text { nephrotoxic, } \\
\text { Abortifacient }\end{array}$ & Dill apiole & $\begin{array}{c}\text { dill apiole }(20.7-52.5 \%), \\
\text { d-limonene }(5.9-45.0 \%), \\
(+) \text {-carvone }(17.4-23.1 \%) \\
\text { (E)-dihydrocarvone }(4.2-16.6 \%) \\
\alpha \text {-phellandrene }(\operatorname{tr}-6.5 \%), \text { and } \\
\text { (Z)-dihydrocarvone }(0.8-5.2 \%)\end{array}$ & - & [32] \\
\hline $\begin{array}{c}\text { Lanyana (African } \\
\text { wormwood) }\end{array}$ & $\begin{array}{c}\text { Artemisia afra Jacq. ex } \\
\text { Willd. }\end{array}$ & Asteraceae & $\begin{array}{l}\text { Leaves and } \\
\text { stems }\end{array}$ & neurotoxic & Thujone & $\begin{array}{c}\alpha \text {-thujone }(22.5 \%), \\
(E) \text {-chrysanthenyl acetate }(19.2 \%), \\
1,8 \text {-cineole }(19.1 \%), \text { camphor } \\
(11.0 \%) \text {, and } \beta \text {-thujone }(8.9 \%)\end{array}$ & - & {$[22]$} \\
\hline Lemon basil & Ocimum $\times$ africanum Lour. & Lamiaceae & Leaves & Teratogenicity & Citral & $\begin{array}{c}\text { geranial }(23.3-25.1 \%), \text { neral } \\
(16.0-17.1 \%), \text { nerol }(13.0-15.3 \%) \\
\text { linalool }(5.0-7.8 \%), \text { and } \\
(E)-\alpha \text {-bisabolene }(5.3-6.2 \%)\end{array}$ & $\begin{array}{c}99 \mathrm{mg} / \text { day based } \\
\text { on } 42.2 \% \text { citral } \\
\text { content }\end{array}$ & {$[22]$} \\
\hline $\begin{array}{l}\text { Lemon leaf (lemon } \\
\text { petitgrain) }\end{array}$ & $\begin{array}{c}\text { Citrus } \times \text { limon } \mathrm{L} . \\
\text { (synonym: Citrus limonum } \\
\text { Risso) }\end{array}$ & Rutaceae & Leaves & Teratogenicity & Citral & $\begin{array}{c}\text { geranial }(10.9-39.0 \%), \text { limonene } \\
(8.1-30.7 \%), \text { neral }(6.5-25.3 \%), \\
\text { geraniol }(0.5-15.0 \%), \beta \text {-pinene } \\
(3.5-13.6 \%), \text { neryl acetate } \\
(3.7-7.4 \%), \text { nerol }(1.3-7.4 \%), \\
\alpha \text {-terpinyl acetate }(\operatorname{tr}-7.3 \%), \text { and } \\
\text { linalyl acetate }(\operatorname{tr}-6.5 \%)\end{array}$ & $\begin{array}{l}84 \mathrm{mg} \text { based on } \\
50 \% \text { citral content }\end{array}$ & [32] \\
\hline $\begin{array}{c}\text { Lemon Myrtle } \\
\text { (lemon ironwood } \\
\text { or sweet verbena } \\
\text { tree) }\end{array}$ & $\begin{array}{c}\text { Backhousia citriodora F. } \\
\text { Muell. }\end{array}$ & Myrtaceae & Leaves & Teratogenicity & Citral & $\begin{array}{c}\text { geranial }(46.1-60.7 \%) \text { and neral } \\
\qquad(32.0-40.9 \%)\end{array}$ & $46 \mathrm{mg} /$ day & [42] \\
\hline
\end{tabular}


Table 1. Cont.

\begin{tabular}{|c|c|c|c|c|c|c|c|c|}
\hline EO & Botanical Name & Family & Part Used & Hazard(s) & $\begin{array}{c}\text { Toxic } \\
\text { Component(s) }\end{array}$ & Oil Composition & $\begin{array}{l}\text { Maximum Oral } \\
\text { Dose in } \\
\text { Pregnancy [22] }\end{array}$ & Ref. \\
\hline Lemon Thyme & $\begin{array}{l}\text { Thymus citriodorus (Pers.) } \\
\text { Schreb. (Synonyms: } \\
\text { Thymus lanuginosus Mill. } \\
\text { var. citriodorum Pers., } \\
\text { Thymus serpyllum var. } \\
\text { citriodorus (Hort.), Thymus } \\
\text { serpyllum L. var. vulgaris } \\
\text { Benth.); a cross between } \\
\text { Thymus vulgaris and } \\
\text { Thymus pulegioides. }\end{array}$ & Lamiaceae & $\begin{array}{l}\text { Aerial } \\
\text { parts }\end{array}$ & Teratogenicity & Citral & $\begin{array}{c}\text { geraniol }(39.2 \%), \text { carvacrol } \\
(15.4 \%) \text {, geranial }(9.2 \%) \text { and neral } \\
(7.1 \%)\end{array}$ & $\begin{array}{c}258 \mathrm{mg} / \text { day based } \\
\text { on } 16.3 \% \text { citral } \\
\text { content }\end{array}$ & [43] \\
\hline Lemongrass & $\begin{array}{l}\text { Cymbopogon flexuosus } \\
\text { Nees ex Steud. (synonym.: } \\
\text { Andropogon flexuosus Nees } \\
\text { ex Steud.) (East Indian) } \\
\text { and Cymbopogon citratus } \\
\text { DC (synonym: } \\
\text { Andropogon citratus DC) } \\
\text { (West Indian) }\end{array}$ & Poaceae & Leaves & Teratogenicity & Citral & $\begin{array}{ll}\text { - } & \text { East Indian lemongrass: } \\
\text { geranial }(45.1-54.5 \%) \text { and } \\
\text { neral }(30.1-36.1 \%) . \\
\text { - West Indian lemongrass: } \\
\text { geranial }(36.7-55.9 \%), \text { neral } \\
(25.0-35.2 \%), \beta-m y r c e n e \\
(5.6-19.2 \%), \text { geraniol } \\
(0-6.7 \%), \text { and limonene } \\
\text { oxide }(0-6.4 \%)\end{array}$ & $\begin{array}{c}46 \mathrm{mg} / \text { day based } \\
\text { on } 90 \% \text { citral } \\
\text { content }\end{array}$ & $\begin{array}{c}{[41,44,} \\
45]\end{array}$ \\
\hline $\begin{array}{l}\text { Lemon-scented tea } \\
\text { tree (lemon tea } \\
\text { tree) }\end{array}$ & $\begin{array}{l}\text { Leptospermum petersonii F. } \\
\text { M. Bailey (synonym: } \\
\text { Leptospermum citratum } \\
\text { Chall., Cheel and Penf.; } \\
\text { Leptospermum liversidgei } \\
\text { R.T. Baker and H. G. } \\
\text { Smith) }\end{array}$ & Myrtaceae & $\begin{array}{l}\text { Aerial } \\
\text { parts }\end{array}$ & Teratogenicity & Citral & $\begin{array}{c}\text { geranial }(45.4 \%), \text { neral }(31.3 \%), \\
\alpha \text {-pinene }(12.3 \%), \text { and citronellal } \\
(6.8 \%)\end{array}$ & $\begin{array}{c}54 \mathrm{mg} / \text { day based } \\
\text { on } 77 \% \text { citral } \\
\text { content }\end{array}$ & [46] \\
\hline $\begin{array}{l}\text { Lesser Calamint } \\
\text { (Cuckoo flower, } \\
\text { field balm, and } \\
\text { nepitella) }\end{array}$ & $\begin{array}{l}\text { Calamintha nepeta L. subsp. } \\
\text { glandulosa Req. (synonym: } \\
\text { Calamintha officinalis } \\
\text { Moench.) }\end{array}$ & Lamiaceae & $\begin{array}{l}\text { Aerial } \\
\text { parts }\end{array}$ & $\begin{array}{l}\text { Abortifacient; } \\
\text { hepatotoxicity }\end{array}$ & Pulegone & $\begin{array}{c}(1 R)-(+)-\beta \text {-pulegone }(17.6-76.1 \%), \\
\text { menthone }(7.0-55.8 \%) \text {, piperitone } \\
\text { oxide }(0-12.4 \%), \text { piperitone } \\
(0-7.4 \%), \text { piperitenone }(0-7.3 \%), \\
\text { limonene }(0.6-7.2 \%), \text { and } \\
\text { terpinen-4-ol }(0-6.8 \%) .\end{array}$ & - & {$[47]$} \\
\hline
\end{tabular}


Table 1. Cont.

\begin{tabular}{|c|c|c|c|c|c|c|c|c|}
\hline EO & Botanical Name & Family & Part Used & Hazard(s) & $\begin{array}{c}\text { Toxic } \\
\text { Component(s) }\end{array}$ & Oil Composition & $\begin{array}{l}\text { Maximum Oral } \\
\text { Dose in } \\
\text { Pregnancy [22] }\end{array}$ & Ref. \\
\hline $\begin{array}{c}\text { May chang } \\
\text { (Pheasant pepper } \\
\text { tree) }\end{array}$ & $\begin{array}{c}\text { Litsea cubeba (Lour.) Pers. } \\
\text { (synonyms: Litsea citrata } \\
\text { Blume, Laurus cubeba } \\
\text { Lour.) }\end{array}$ & Lauraceae & Fruits & Teratogenicity & Citral & $\begin{array}{c}\text { geranial }(37.9-40.6 \%), \text { neral } \\
(25.5-33.8 \%), \text { limonene } \\
(8.4-22.6 \%), \text { and methyl } \\
\text { heptenone }(0.5-4.4 \%)\end{array}$ & $\begin{array}{l}56 \mathrm{mg} / \text { day based } \\
\text { on } 74 \% \text { citral } \\
\text { content }\end{array}$ & {$[41,48$} \\
\hline $\begin{array}{l}\text { Melissa (lemon } \\
\text { balm) }\end{array}$ & Melissa officinalis L. & Lamiaceae & $\begin{array}{c}\text { Fresh } \\
\text { aerial parts }\end{array}$ & Teratogenicity & Citral & $\begin{array}{c}\text { geranial }(12.5-38.3 \%), \text { neral } \\
(9.7-26.1 \%), \beta \text {-caryophyllene } \\
(0.3-19.1 \%), \text { citronellal } \\
(4.5-13.3 \%), \text { germacrene D } \\
(0-13.0 \%), \text { caryophyllene oxide } \\
(0.8-10.0 \%), \text { and geraniol } \\
(1.0-8.1 \%)\end{array}$ & $\begin{array}{l}65 \mathrm{mg} / \text { day based } \\
\text { on } 64 \% \text { citral } \\
\text { content }\end{array}$ & {$[37,48$} \\
\hline $\begin{array}{c}\text { Mugwort } \\
\text { (chrysanthenyl } \\
\text { acetate CT) }\end{array}$ & Artemisia vulgaris $\mathrm{L}$. & Asteraceae & $\begin{array}{l}\text { Aerial } \\
\text { parts }\end{array}$ & slightly neurotoxic & Thujone & $\begin{array}{c}\text { chrysanthenyl acetate } \\
(31.7-32.8 \%) \text { and germacrene D } \\
(12.1-15.9 \%)\end{array}$ & - & [22] \\
\hline $\begin{array}{c}\text { Mugwort or } \\
\text { Indian wormwood } \\
\text { oil (cam- } \\
\text { phor/thujone } \\
\text { CT) }\end{array}$ & Artemisia vulgaris L. & Asteraceae & $\begin{array}{l}\text { Aerial } \\
\text { parts of } \\
\text { flowering } \\
\text { plant }\end{array}$ & Slightly neurotoxic & Thujone & $\begin{array}{c}\text { camphor }(20.8 \%), \text { artemisia } \\
\text { alcohol }(15.3 \%), \alpha \text {-thujone } \\
(11.4 \%), \beta \text {-caryophyllene }(10.6 \%), \\
\text { isoborneol }(9.3 \%), 1,8 \text {-cineole } \\
(9.0 \%), \text { and sabinene }(6.1 \%)\end{array}$ & - & [49] \\
\hline $\begin{array}{l}\text { Myrrh (Somalian } \\
\text { myrrh) }\end{array}$ & $\begin{array}{l}\text { Commiphora myrrha (Nees) } \\
\text { Engl. (synonym: } \\
\text { Commiphora molmol Engl.) }\end{array}$ & Burseraceae & $\begin{array}{c}\text { Dried gum } \\
\text { oleoresin }\end{array}$ & $\begin{array}{c}\text { Fetotoxic, } \\
\text { anti-angiogenic }\end{array}$ & $\begin{array}{l}\beta \text {-Elemene and } \\
\text { furanodiene }\end{array}$ & $\begin{array}{c}\text { furanoeudesma-1,3-diene }(34.0 \%), \\
\text { furanodiene }(19.7 \%) \text {, lindestrene } \\
(12.0 \%) \text {, and } \beta \text {-elemene }(8.7 \%)\end{array}$ & - & {$[50]$} \\
\hline $\begin{array}{l}\text { Nasturtium } \\
\text { (Indian cress) } \\
\text { absolute }\end{array}$ & Tropaeolum majus L. & Tropaeolaceae & Flowers & fetal toxicity & $\begin{array}{l}\text { Benzyl cyanide, } \\
\text { benzyl } \\
\text { isothiocyanate }\end{array}$ & $\begin{array}{c}\text { benzyl isothiocyanate }(72.3 \%) \\
\text { unidentified nitrogen compound } \\
(16.0 \%) \text {, and benzyl cyanide } \\
(2.0 \%)\end{array}$ & - & [22] \\
\hline
\end{tabular}


Table 1. Cont.

\begin{tabular}{|c|c|c|c|c|c|c|c|c|}
\hline EO & Botanical Name & Family & Part Used & Hazard(s) & $\begin{array}{c}\text { Toxic } \\
\text { Component(s) }\end{array}$ & Oil Composition & $\begin{array}{l}\text { Maximum Oral } \\
\text { Dose in } \\
\text { Pregnancy [22] }\end{array}$ & Ref. \\
\hline Nutmeg & $\begin{array}{l}\text { Myristica fragrans Houtt } \\
\text { (Synonyms: Myristica } \\
\text { officinalis L. fil., Myristica } \\
\text { moschata Thunb., Myristica } \\
\text { aromatica O. Schwartz, } \\
\text { and Myristica amboinensis } \\
\text { Gand.) }\end{array}$ & Myristicaceae & Kernels & $\begin{array}{c}\text { Potentially } \\
\text { carcinogenic; } \\
\text { reduced fertility }\end{array}$ & $\begin{array}{c}\text { Safrole, } \\
\text { methyleugenol, } \\
\text { myristicin }\end{array}$ & $\begin{array}{l}\text { East Indian EO: sabinene } \\
(14.0-44.1 \%), \alpha \text {-pinene } \\
(18.0-26.5 \%), \beta \text {-pinene } \\
(8.7-17.7 \%), \text { myristicin } \\
(3.3-13.5 \%), \text { terpinen-4-ol } \\
(1.0-10.9 \%), \gamma \text {-terpinene } \\
(1.3-7.7 \%), \text { linalool } \\
(0.2-7.4 \%), \text { limonene } \\
(2.0-7.0 \%), \alpha \text {-phellandrene } \\
(0.4-5.8 \%) \text { and } \alpha \text {-terpinene } \\
(0.1-5.2 \%) . \\
\text { West Indian EO: sabinene } \\
(42.0-57.0 \%), \alpha \text {-pinene } \\
(1.6-12.6 \%), \beta \text {-pinene } \\
(7.8-12.1 \%) \text { and } \\
\text { terpinen-4-ol }(3.0-6.4 \%)\end{array}$ & - & {$[32,51]$} \\
\hline $\begin{array}{l}\text { Orange Champaca } \\
\text { (golden champa, } \\
\text { champak) absolute }\end{array}$ & Michelia champaca L. & Magnoliaceae & Flowers & Toxic & 2-Phenylethanol & $\begin{array}{c}\text { 2-phenylethanol (25.0-34.0\%), } \\
\text { methyl linoleate }(10.0-18.0 \%) \\
\text { indole (2.9-12.0\%), methyl } \\
\text { anthranilate }(2.1-9.0 \%), \text { and } \\
\text { methyl benzoate }(1.0-5.0 \%)\end{array}$ & - & [52] \\
\hline Oregano & $\begin{array}{c}\text { Origanum onites L. } \\
\text { (synonym: Origanum } \\
\text { smyrnaeum L.); Origanum } \\
\text { vulgare L. subsp. hirtum } \\
\text { (Link) Ietswaart } \\
\text { (synonym: Origanum } \\
\text { compactum, Origanum } \\
\text { hirtum Link); and Thymbra } \\
\text { capitata (L.) Cav. } \\
\text { (synonym: Thymus } \\
\text { capitatus L., Coridothymus } \\
\text { capitatus L., Satureja } \\
\text { capitata L.) }\end{array}$ & Lamiaceae & $\begin{array}{l}\text { Dried } \\
\text { aerial parts } \\
\text { of } \\
\text { flowering } \\
\text { plant }\end{array}$ & embryotoxic & Not identified & 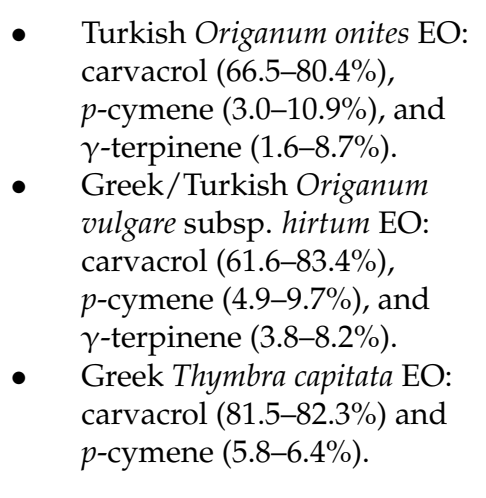 & - & [53-56] \\
\hline
\end{tabular}


Table 1. Cont

\begin{tabular}{|c|c|c|c|c|c|c|c|c|}
\hline EO & Botanical Name & Family & Part Used & Hazard(s) & $\begin{array}{c}\text { Toxic } \\
\text { Component(s) }\end{array}$ & Oil Composition & $\begin{array}{l}\text { Maximum Oral } \\
\text { Dose in } \\
\text { Pregnancy [22] }\end{array}$ & Ref. \\
\hline Parsley leaf & $\begin{array}{c}\text { Petroselinum crispum Mill } \\
\text { (synonym: } P \text { sativum } \\
\text { Hoffm., and } P . \\
\text { hortenseauct) }\end{array}$ & Apiaceae & Leaves & abortifacient & Parsley apiole & $\begin{array}{c}\text { Egyptian parsley: } \\
p \text {-mentha-1,3,8-triene }(6.2-45.2 \%), \\
\beta \text {-myrcene }(7.8-23.8 \%), \\
\beta \text {-phellandrene }(6.7-19.5 \%), \\
\text { myristicin }(1.9-8.8 \%), \alpha \text {-pinene } \\
(6.9-7.6 \%), \text { terpinolene }(2.8-6.6 \%), \\
\text { limonene }(3.3-5.4 \%), \\
\alpha \text { - } p \text {-dimethylstyrene }(2.7-5.4 \%), \\
\text { and dill apiole }(0.2-5.2 \%)\end{array}$ & - & {$[22,57]$} \\
\hline Parsley seed & Petroselinum crispum Mill & Apiaceae & Seeds & abortifacient & Parsley apiole & $\begin{array}{c}\text { parsley apiole }(11.3-67.5 \%), \\
\text { myristicin }(0.7-37.9 \%), \\
\text { allyltetramethoxybenzene } \\
(0.6-29.0 \%), \alpha \text {-pinene }(8.3-16.9 \%), \\
\beta-\text { pinene }(5.4-10.7 \%), \text { and } \\
\text { elemicin }(0-8.8 \%)\end{array}$ & - & {$[58]$} \\
\hline Plectranthus & $\begin{array}{c}\text { Plectranthus fruticosus } \\
\text { L'Hérit }\end{array}$ & Lamiaceae & Leaves & $\begin{array}{l}\text { embryotoxic, } \\
\text { fetotoxic, } \\
\text { teratogenic and } \\
\text { abortifacient }\end{array}$ & Sabinyl acetate & sabinyl acetate $(>60.0 \%)$ & - & [59] \\
\hline
\end{tabular}


Table 1. Cont.

\begin{tabular}{|c|c|c|c|c|c|c|c|c|}
\hline EO & Botanical Name & Family & Part Used & Hazard(s) & $\begin{array}{c}\text { Toxic } \\
\text { Component(s) }\end{array}$ & Oil Composition & $\begin{array}{l}\text { Maximum Oral } \\
\text { Dose in } \\
\text { Pregnancy [22] }\end{array}$ & Ref. \\
\hline Rue & $\begin{array}{l}\text { Ruta graveolens L. and } \\
\text { Ruta montana Mill }\end{array}$ & Rutaceae & $\begin{array}{c}\text { Aerial } \\
\text { parts }\end{array}$ & abortifacient & Not identified & $\begin{array}{l}\text { Egyptian R. graveolens EO: } \\
\text { 2-undecanone }(49.2 \%), \\
\text { 2-nonanone }(24.7 \%), \text { and } \\
\text { 2-nonyl acetate }(6.2 \%) \\
\text { Italian } R \text {. graveolens EO: } \\
\text { 2-undecanone }(46.8 \%) \text { and } \\
\text { 2-nonanone }(18.8 \%) \\
\text { Non-volatiles: angelicin } \\
(0.043 \%), \text { methoxsalen } \\
(0.032 \%), \text { isopimpinellin } \\
(0.02 \%), \text { bergapten }(0.018 \%), \\
\text { and psoralen }(0.015 \%)\end{array}$ & - & {$[60-62]$} \\
\hline Savin & Juniperus sabina L. & Cupressaceae & $\begin{array}{l}\text { Leaves and } \\
\text { terminal } \\
\text { branches }\end{array}$ & $\begin{array}{l}\text { embryo-fetotoxic, } \\
\text { abortifacient and } \\
\text { hepatotoxic }\end{array}$ & $\begin{array}{l}\text { trans-Sabinyl } \\
\text { acetate }\end{array}$ & $\begin{array}{c}\text { trans-sabinyl acetate }(19.1-53.1 \%) \\
\text { sabinene }(18.3-40.8 \%), \text { and } \\
\text { elemol }(\operatorname{tr}-7.0 \%)\end{array}$ & - & [23] \\
\hline Sea Wormwood & $\begin{array}{c}\text { Artemisia maritima L. } \\
\text { (synonyms: Artemisia } \\
\text { contra Willd. ex Spreng., } \\
\text { Artemisia lercheana Kar. } \\
\text { and Kir., Artemisia salina } \\
\text { Willd., Seriphidium } \\
\text { maritimum (L.) Poljakov) }\end{array}$ & Asteraceae & $\begin{array}{l}\text { Leaves and } \\
\text { flowering } \\
\text { tops }\end{array}$ & neurotoxic & Thujone & $\begin{array}{c}\alpha \text {-thujone }(63.3 \%) \text {, sabinene } \\
(7.8 \%) \text { and } 1,8 \text {-cineole }(6.5 \%)\end{array}$ & - & [63] \\
\hline $\begin{array}{l}\text { Spanish Lavender } \\
\text { (French lavender } \\
\text { or maritime } \\
\text { lavender) }\end{array}$ & $\begin{array}{l}\text { Lavandula stoechas L. ssp. } \\
\text { stoechas }\end{array}$ & Lamiaceae & $\begin{array}{l}\text { Flowering } \\
\text { tops }\end{array}$ & neurotoxic & Camphor & $\begin{array}{c}\text { camphor }(16.4-56.2 \%) \\
\text { (+)-fenchone }(14.9-49.1 \%) \\
\text { 1,8-cineole }(3.6-14.5 \%), \alpha \text {-pinene } \\
(3.4-4.5 \%), \text { and camphene } \\
(2.8-5.5 \%)\end{array}$ & - & {$[64]$} \\
\hline
\end{tabular}


Table 1. Cont

\begin{tabular}{|c|c|c|c|c|c|c|c|c|}
\hline EO & Botanical Name & Family & Part Used & Hazard(s) & $\begin{array}{c}\text { Toxic } \\
\text { Component(s) }\end{array}$ & Oil Composition & $\begin{array}{l}\text { Maximum Oral } \\
\text { Dose in } \\
\text { Pregnancy [22] }\end{array}$ & Ref. \\
\hline $\begin{array}{l}\text { Spanish Sage } \\
\text { (lavender sage) }\end{array}$ & $\begin{array}{l}\text { Salvia lavandulifolia Vahl } \\
\text { (synonym: Salvia } \\
\text { hispanorum Lag) }\end{array}$ & Lamiaceae & $\begin{array}{l}\text { Flowering } \\
\text { tops }\end{array}$ & abortifacient & Sabinyl acetate & $\begin{array}{l}\text { Flowering tops oil: } \\
\text { 1,8-cineole (12.0-40.3\%), } \\
\text { camphor (12.9-36.1\%), } \\
\alpha \text {-terpinyl acetate } \\
(0.5-15.5 \%), \text { linalool } \\
(0.2-11.2 \%), \alpha \text {-pinene } \\
(4.7-10.9 \%), \text { camphene } \\
(4.6-10.6 \%), \beta \text {-pinene } \\
(3.3-7.3 \%),(Z) \text {-sabinyl } \\
\text { acetate }(0.5-9.0 \%), \text { borneol } \\
(1.5-6.4 \%), \text { linalyl acetate } \\
(0.1-5.8 \%), \text { and limonene } \\
(2.4-5.0 \%) \\
\text { Aerial parts oil (steam } \\
\text { distilled): 1,8-cineole } \\
(21.4-33.8 \%), \alpha \text {-pinene } \\
(10.5-17.5 \%), \beta \text {-pinene } \\
(6.0-17.3 \%), \text { limonene } \\
(5.6-10.4 \%), \text { camphor } \\
(6.1-9.4 \%), \\
\text { trans-caryophyllene } \\
(4.0-8.5 \%), \text { and myrcene } \\
(\text { tr-10.0\%). }\end{array}$ & - & {$[23,65]$} \\
\hline Star anise & Illicium verum J.D. Hook. & Illiciaceae & Fruits & $\begin{array}{l}\text { reproductive } \\
\text { hormone } \\
\text { modulation }\end{array}$ & (E)-Anethole & $\begin{array}{c}(E) \text {-anethole }(71.2-91.8 \%) \\
\text { foeniculin }(0.5-14.6 \%), \text { estragole } \\
(0.3-6.6 \%) \text {, and } d \text {-limonene } \\
(0.7-5.0 \%)\end{array}$ & - & [23] \\
\hline $\begin{array}{l}\text { Sweet Birch (black } \\
\text { birch or southern } \\
\text { birch) }\end{array}$ & Betula lenta $\mathrm{L}$. & Betulaceae & Bark & $\begin{array}{l}\text { reproductively } \\
\text { toxic }\end{array}$ & $\begin{array}{l}\text { Methyl salicylate } \\
\text { and ethyl } \\
\text { salicylate }\end{array}$ & $\begin{array}{l}\text { methyl salicylate }(90.4 \%) \text { and } \\
\text { ethyl salicylate }(5.5 \%)\end{array}$ & - & [22] \\
\hline Sweet Fennel & Foeniculum vulgare Mill. & Apiaceae & Seeds & $\begin{array}{l}\text { Reproductive } \\
\text { hormone } \\
\text { modulation }\end{array}$ & (E)-Anethole & $\begin{array}{c}\text { (E)-anethole }(58.1-92.5 \%) \\
d \text {-limonene }(0.2-21.0 \%), \text { fenchone } \\
(0.2-8.0 \%), \text { and estragole } \\
(1.1-4.8 \%)\end{array}$ & - & {$[23,24]$} \\
\hline
\end{tabular}


Table 1. Cont.

\begin{tabular}{|c|c|c|c|c|c|c|c|c|}
\hline EO & Botanical Name & Family & Part Used & Hazard(s) & $\begin{array}{c}\text { Toxic } \\
\text { Component(s) }\end{array}$ & Oil Composition & $\begin{array}{l}\text { Maximum Oral } \\
\text { Dose in } \\
\text { Pregnancy [22] }\end{array}$ & Ref. \\
\hline Tansy & $\begin{array}{c}\text { Tanacetum vulgare } \mathrm{L} . \\
\text { (synonyms: } \\
\text { Chrysanthemum tanacetum } \\
\text { Karsch, and } \\
\text { Chrysanthemum vulgare L.) }\end{array}$ & Asteraceae & $\begin{array}{c}\text { Aerial } \\
\text { parts }\end{array}$ & neurotoxic & Thujone & $\begin{array}{l}\beta \text {-thujone }(45.2 \%) \text {, artemisia } \\
\text { ketone }(10.5 \%), \text { borneol }(7.8 \%), \\
\text { and bornyl acetate }(7.7 \%)\end{array}$ & - & [22] \\
\hline $\begin{array}{l}\text { Thuja (cedar leaf, } \\
\text { white cedar, } \\
\text { eastern white } \\
\text { cedar, eastern } \\
\text { arborvitae, or } \\
\text { swamp cedar) }\end{array}$ & Thuja occidentalis L. & Cupressaceae & $\begin{array}{l}\text { Fresh } \\
\text { leaves and } \\
\text { terminal } \\
\text { branches }\end{array}$ & neurotoxic & Thujone & $\begin{array}{c}\alpha \text {-thujone }(48.7-51.5 \%) \text {, fenchone } \\
(12.2-12.8 \%) \text { and } \beta \text {-thujone } \\
(7.9-9.9 \%)\end{array}$ & - & {$[22,66]$} \\
\hline $\begin{array}{l}\text { Verbena (lemon } \\
\text { verbena) }\end{array}$ & $\begin{array}{c}\text { Aloysia triphylla L'Hérit } \\
\text { (Synonyms: Aloysia } \\
\text { citriodora Ortega ex Pers., } \\
\text { Lippia citriodora Ortega ex } \\
\text { Pers., and Lippia triphylla } \\
\text { L'Hérit) }\end{array}$ & Verbenaceae & Leaves & Teratogenicity & Citral & $\begin{array}{c}\text { geranial }(29.5-38.3 \%) \text {, neral } \\
(22.9-29.6 \%) \text {, and limonene } \\
(5.7-15.4 \%)\end{array}$ & $\begin{array}{l}61 \mathrm{mg} / \text { day based } \\
\text { on } 68 \% \text { citral } \\
\text { content }\end{array}$ & [67] \\
\hline $\begin{array}{l}\text { Western red cedar } \\
\text { (pacific thuja or } \\
\text { western } \\
\text { arborvitae) }\end{array}$ & $\begin{array}{c}\text { Thuja plicata Donn ex D. } \\
\text { Don }\end{array}$ & Cupressaceae & $\begin{array}{l}\text { Needles } \\
\text { (leaves) }\end{array}$ & neurotoxic & Thujones & $\begin{array}{c}\alpha \text {-thujone }(63.5-84.0 \%), \\
\beta \text {-thujone }(4.9-15.2 \%), \text { and } \\
\text { sabinene }(1.1-8.8 \%)\end{array}$ & - & [68] \\
\hline $\begin{array}{l}\text { White Wormwood } \\
\text { (armoise or desert } \\
\text { wormwood) } \alpha- \\
\text { thujone/camphor } \\
\text { chemotype }\end{array}$ & Artemisia herba-alba Asso & Asteraceae & $\begin{array}{l}\text { Leaves and } \\
\text { flowering } \\
\text { tops }\end{array}$ & neurotoxic & $\begin{array}{l}\text { Thujones; } \\
\text { camphor }\end{array}$ & $\begin{array}{c}\text { camphor }(34.0-55.0 \%), \alpha \text {-thujone } \\
(25.7-36.8 \%), \beta \text {-thujone } \\
(2.0-9.0 \%), \text { camphene }(0.5-9.0 \%) \\
\text { and } 1,8 \text {-cineole }(1.5-8.0 \%)\end{array}$ & - & $\begin{array}{c}{[32,41,} \\
69]\end{array}$ \\
\hline Wintergreen & $\begin{array}{c}\text { Gaultheria fragrantissima } \\
\text { Wall. and Gaultheria } \\
\text { procumbens L. }\end{array}$ & Ericaceae & Leaves & $\begin{array}{l}\text { high doses are } \\
\text { teratogenic }\end{array}$ & Methyl salicylate & $\begin{array}{l}\text { - Nepalese G. fragrantissima: } \\
\text { methyl salicylate } \\
\text { (97.0-99.5\%) } \\
\text { Chinese G. procumbens: } \\
\text { methyl salicylate } \\
(96.0-99.0 \%)\end{array}$ & - & [22] \\
\hline
\end{tabular}


Table 1. Cont

\begin{tabular}{|c|c|c|c|c|c|c|c|c|}
\hline EO & Botanical Name & Family & Part Used & Hazard(s) & $\begin{array}{c}\text { Toxic } \\
\text { Component(s) }\end{array}$ & Oil Composition & $\begin{array}{l}\text { Maximum Oral } \\
\text { Dose in } \\
\text { Pregnancy [22] }\end{array}$ & Ref. \\
\hline $\begin{array}{l}\text { Wormwood } \\
\text { (Absinthe) }\end{array}$ & Artemisia absinthium L. & Asteraceae & $\begin{array}{l}\text { Leaves and } \\
\text { flowering } \\
\text { tops }\end{array}$ & $\begin{array}{l}\text { embryo- } \\
\text { fetotoxicity; } \\
\text { abortifacient }\end{array}$ & $\begin{array}{c}\text { Sabinyl acetate; } \\
\text { Thujone }\end{array}$ & $\begin{array}{l}\text { - } \quad \beta \text {-thujone chemotype: } \\
\beta \text {-thujone (33.1-59.9\%), and } \\
\text { trans-sabinyl acetate } \\
(18.1-32.8 \%) \\
\beta \text {-thujone } /(Z) \text {-epoxy- } \\
\text { ocimene chemotype: } \\
\text { (Z)-epoxy- } \alpha \text {-ocimene } \\
(24.2-28.9 \%), \beta \text {-thujone } \\
(20.9-21.7 \%), \text { and } \\
\text { chrysanthendiol (5.3-6.6\%) } \\
\text { (Z)-epoxy-ocimene } \\
\text { chemotype: } \\
\text { (Z)-epoxy- } \alpha \text {-ocimene } \\
\text { (25.7-42.2\%), chrysanthenyl } \\
\text { acetate }(9.9-15.6 \%), \text { and } \\
\text { sabinyl acetate }(0.3-7.4 \%) \\
\text { sabinyl acetate chemotype: } \\
\text { sabinyl acetate }(31.5 \%), \\
\text { neryl isovalerate }(9.1 \%), \\
\text { neryl butyrate }(7.9 \%), \text { and } \\
\text { chrysanthenyl acetate (5.8\%) }\end{array}$ & - & {$[23,39]$} \\
\hline $\begin{array}{l}\text { Zedoary (white } \\
\text { turmeric, hidden } \\
\text { ginger) }\end{array}$ & Curcuma zedoaria Roscoe & Zingiberaceae & Rhizome & $\begin{array}{c}\text { antifertility; } \\
\text { embryotoxicity, } \\
\text { antigestational } \\
\text { and abortifacient }\end{array}$ & Not identified & $\begin{array}{c}\text { epicurzerene }(19.0-46.6 \%), \\
\text { curzerene }(10.4 \%), \text { curdione } \\
(7.0-19.6 \%), \text { curzerenone } \\
(22.3-31.6 \%), \\
\text { debromofiliforminol }(31.5 \%), \\
\text { 1,8-cineole }(18.5-40.8 \%), \\
\beta \text {-sesquiphellandrene }(21.5 \%), \\
\text { p-cymene }(18.4 \%), \text { curcumenene } \\
\text { (18.7\%), and } \alpha \text {-phellandrene } \\
(14.9 \%)\end{array}$ & - & $\begin{array}{c}{[66,70-} \\
73]\end{array}$ \\
\hline
\end{tabular}




\section{Anethole-Rich Essential Oils}

(E)-Anethole is a phenylpropenoid ether, found in anise (Pimpinella anisum L.) oil (75.2-96.1\%) [23,24], aniseed myrtle (Syzygium anisatum (Vickery) Craven and Biffin) oil (95.0\%) [25], sweet fennel (Foeniculum vulgare Mill.) oil (58.1-92.5\%) [23,24], star anise (Illicium verum Hook. f.) oil (71.2-91.8\%) [23], bitter fennel (Foeniculum vulgare Mill. subsp. capillaceum Gilib.) oil (52.5-84.3\%) [23], and betel oil (Piper betle L.) (0-7.8\%) [22]. (Z)Anethole, an isomer of $(E)$-anethole, is found in sweet fennel oil $(\operatorname{tr}-0.7 \%)$, anise $(\operatorname{tr}-0.5 \%)$, star anise $(\operatorname{tr}-0.4 \%)$, and bitter fennel (tr-0.2\%) [22]. (Z)-Anethole is considerably more toxic than the common isomer (E)-anethole [74].

(E)-Anethole was reported to have anti-hypernociceptive, anticancer, antiplatelet, anti-inflammatory, and anesthetic properties [75-77]. However, administration of $(E)$ anethole-rich EOs (by any route) should be avoided in pregnancy, breastfeeding, and estrogen-dependent cancers. Additionally, internal use of $(E)$-anethole-rich EOs is not advisable in childbirth due to its antiplatelet aggregation activity. There is enough evidence for the estrogenic action of $(E)$-anethole. $(E)$-Anethole was estrogenic in yeast assays (in vitro) [1,78]. It was reported to bind to estrogen receptors in engineered yeast cells [1]. In humans, sweet fennel tea (rich in (E)-anethole) was estrogenic in vivo [79]. A notable increase in uterine weight was observed in immature female rats following $(E)$-anethole treatment $(80 \mathrm{mg} / \mathrm{kg} /$ day for 3 days), confirming its estrogenic effect [80]. (E)-Anethole showed an anti-implantation effect in pregnant rats. Oral administration of $(E)$-anethole (50, 70, and $80 \mathrm{mg} / \mathrm{kg}$ on gestational days 1-10) to pregnant albino Charles Foster rats caused a dose-dependent reduction in implantation as a result of a disruption of hormonal balance [80]. It is worth mentioning that both mice and humans can metabolize (E)-anethole in a similar way, while rats metabolize it differently [81]. A metabolite of anethole, anethole$1^{\prime}, 2^{\prime}$-epoxide, was carcinogenic and caused the formation of hepatomas and papillomas in mice [82].

Anethole-rich essential oils such as aniseed, star anise, bitter fennel, sweet fennel, and aniseed myrtle are estrogenic in one or more in vitro assays and may cause reproductive hormone modulation [1,78]. These oils are hepatotoxic due to their high $(E)$-anethole content. Bitter fennel oil is hepatotoxic due to the metabolite, anethole-1', $2^{\prime}$-epoxide [83]. Like bitter fennel EO, sweet fennel $\mathrm{EO}$ is a reproductive hormonal level modulator, fetotoxic, and hepatotoxic [84]. Sweet fennel tea (containing 1.3-10.0\% of the oil [85]) showed in vivo estrogenic activity in humans and its prolonged use caused premature breast development and significantly higher serum estradiol levels [79]. A sweet fennel oil (with $72 \%(E)-$ anethole, $12.0 \%$ fenchone, and $5 \%$ estragole) was teratogenic at $0.93 \mathrm{mg} / \mathrm{mL}$ and produced about $50 \%$ reduction in differentiated rat embryo limb bud foci. It dose-dependently decreased the intensity of oxytocin or prostaglandin E2-induced uterine contractions ex vivo [86] which is why the use of sweet fennel oil is not advisable during slow-progressing labor. Therefore, consumption of anethole-rich essential oils is unsafe and should be avoided (by any route) during pregnancy, breastfeeding, and in some estrogen-dependent cancers $[87,88]$. These oils are potentially carcinogenic based on their estragole and safrole (minor components) content [89]. (E)-Anethole and estragole interfered with fetoplacental steroidogenesis in a co-culture of human adrenocortical carcinoma cells (H295R) and human placental choriocarcinoma cells (BeWo) cells by increasing hormonal concentrations and altering steroidogenic enzyme activity and expression [90,91].

\section{Methyl Salicylate-Rich Essential Oils}

Methyl salicylate is a phenolic ester that dominates wintergreen (Gaultheria procumbens L.) (96.0-99.5\%) [92] and sweet birch (Betula lenta L.) (90.4\%) oils [22]. Methyl salicylate is largely hydrolyzed into salicylic acid in the liver [93]. Following topical application in humans, methyl salicylate can be transdermally absorbed and converted to salicylic acid in the dermal and subcutaneous tissues [94,95]. Orally taken methyl salicylate is metabolized faster in rats and dogs than in humans which means a higher toxicity in humans [96]. Methyl salicylate poisoning in humans is known to cause fever, nausea, vom- 
iting, CNS excitation, tachycardia, rapid breathing, high blood pressure, respiratory failure, pneumonia, pulmonary edema, convulsions, and coma [97]. Methyl salicylate poisoning in humans has a $50-60 \%$ mortality rate which is a result of cardiovascular collapse and respiratory failure $[98,99]$. Methyl salicylate showed in vitro human estrogen receptor $\alpha$ $(\mathrm{hER} \alpha)$ agonistic activity [100]. Salicylates have been shown to cross the placenta [101] and lead to restricted growth and congenital abnormalities in animal experiments [102]. The fact that superoxide dismutase treatment prevented salicylate-induced malformations in rat embryos suggests that free oxygen radicals play a role in its teratogenic action [103]. Intraperitoneal injection of methyl salicylate $(200$ or $400 \mathrm{mg} / \mathrm{kg}$, pregnant rats on gestational days 9 and 10) dose-dependently reduced the development of the brain, lung, liver, and kidney of the fetus [104]. Methyl salicylate (i.p., 50 or $100 \mu \mathrm{L}$ ) given to female rats on gestational days 10 and 11 resulted in retardation of fetal kidney development. At $100 \mu \mathrm{L}$, maternal weight gain was retarded, the offspring were fewer and smaller, and resorptions and malformations were increased [105]. A single subcutaneous injection of methyl salicylate $(1.5 \mathrm{~mL} / \mathrm{kg}$ on gestation day 7,9 , or 11$)$ to female rats resulted in higher fetal deaths, reduced fetal weight, and cleft palate and tail abnormalities [106]. At 200, 250, or $300 \mathrm{mg} / \mathrm{kg}$ /day (i.p., on gestation days 11-12), methyl salicylate showed teratogenicity and embryotoxicity in pregnant Sprague-Dawley rats [107]. Methyl salicylate increases the occurrence of dilated renal pelvis in the rat fetus and causes a temporary maturation delay in the rat's ability to concentrate urine [107]. In another experiment, several anomalies were observed in the offspring digestive tract, CNS, liver, and skeleton following a single subcutaneous injection of methyl salicylate $(0.1-0.5 \mathrm{~mL}$ on gestational day 9,10 , or 11$)$ in pregnant rats [108]. Subcutaneous administration of methyl salicylate $(400 \mathrm{mg} / \mathrm{kg})$ caused a substantial decrease in plasma calcium levels in pregnant rats and mice which might be linked to the fetal toxicity [109]. In pregnant hamsters, oral or topical methyl salicylate caused neural tube fusion failure in the embryos $[93,110]$. In addition, high oral doses of wintergreen EO were toxic and teratogenic in rats and monkeys [102]. Based on the available information, use of methyl salicylate-rich essential oils or any preparations containing them, by any route, should be avoided during pregnancy and lactation.

\section{4. cis-Sabinyl Acetate-Rich Essential Oils}

cis-Sabinyl acetate is a bicyclic monoterpenoid ester found in Plectranthus (P. fruticosus L'Hér.) (>60.0\%) [59], savin (Juniperus sabina L.) (19.1-53.1\%) [32], wormwood (Artemisia absinthium L., $\beta$-thujone chemotype) (18.1-32.8\%) [32,41,69], wormwood (sabinyl acetate chemotype) (31.5\%) [32,39], wormwood ((Z)-epoxy ocimene chemotype) (0.3-7.4\%) [32,39], and Spanish sage (Salvia lavandulifolia Vahl) $(0-6.6 \%)[32,65]$. It is considered one of the very few toxic essential oil esters. Sabinyl acetate is among the most dangerous constituents in pregnancy since it is maternally toxic and abortifacient [22]. It acts through inhibiting implantation of the embryo [111]. Experiments with sabinyl acetate-rich oils suggest substantial reproductive toxicity risks. Plectranthus oil (60\% sabinyl acetate) is embryotoxic, fetotoxic, teratogenic, and abortifacient [112]. In pregnant rats, oral administration of Plectranthus oil at $0.5,2.5$, or $5.0 \mathrm{mg} / \mathrm{kg}$ on gestational days $6-15$ caused a surge in the rate of resorption and fetal toxicity (abnormally small eyeballs and lack of eyes) [113]. At a dose of $5 \mathrm{mg} / \mathrm{kg}$, Plectranthus oil showed abortifacient and fetotoxic effects in pregnant rats [59]. The strong embryotoxic and fetotoxic action of Plectranthus oil was attributed to its sabinyl acetate content [113]. Subcutaneously injected Plectranthus EO $(15,45$, or $135 \mathrm{mg} / \mathrm{kg})$ to pregnant mice on gestational days 6-15 caused abortion, malformed embryos (kidney and heart defects, skeletal modifications, and lack of eyes), and a rise in resorption in mice [114,115]. Extracts of Plectranthus fruticosus showed antifertility and anti-implantation effects in Wistar rats [112].

Another sabinyl acetate-rich oil is savin oil (Juniperus sabina L.). Savin oil (50\% sabinyl acetate) is embryo-fetotoxic, abortifacient, and hepatotoxic [116]. It can easily cross the placenta and cause abortion [117]. Subcutaneous administration of savin EO to pregnant mice (at 15,45 , or $135 \mathrm{mg} / \mathrm{kg}$ on gestational days 6-15) caused embryotoxicity and sig- 
nificant weight loss [116]. It also inhibited implantation in mice on gestational days $0-4$ but not on gestational days 8-11 suggesting that sabinyl acetate causes abortion [111]. The abortifacient action of the savin plant does not seem to be only due to the oil. An ether extract of Juniperus sabina, prepared after isolating the oil, showed anti-implantation effect in a dose-dependent manner [118]. Since nothing much can be gained from using savin oil, it should not be used either internally or externally. Similarly, juniper berry (Juniperus communis L.) ethanolic extract was clearly abortifacient [119]; however, there is no evidence that the juniper berry EO is abortifacient.

Moreover, Spanish sage oil is a well-known abortifacient. Subcutaneously injected Spanish sage oil fraction (50\% sabinyl acetate) into pregnant mice (at 15, 45, and $135 \mathrm{mg} / \mathrm{kg}$ on gestational days 6-15) caused abortion and maternal toxicity in a dose-dependent manner [115]. Spanish sage oil $(0.01 \mathrm{mg} / \mathrm{mL})$ also induced $\beta$-galactosidase activity in yeast which suggests a possible estrogenic activity [120]. Similarly, wormwood oil is neurotoxic, embryo-fetotoxic, and abortifacient [23,39]. It is particularly hazardous since it carries combined risks from thujones and sabinyl acetate. Since there is no established no observed adverse effect level (NOAEL), it is best to completely avoid sabinyl acetate-rich essential oils and any preparations containing them in pregnancy, especially during the first trimester.

\section{Thujone-Rich Essential Oils}

Thujone is a bicyclic monoterpenoid ketone present as two isomers found together in essential oils: (S)- $\alpha$-thujone and (R)- $\beta$-thujone. (S)- $\alpha$-Thujone is a major component in western red cedar (Thuja plicata Donn ex D. Don) (63.5-84.0\%) [68], génépi (Artemisia genipi Weber ex Stechm.) (79.8\%) [38], sea wormwood (Artemisia maritima L.) (63.3\%) [63], thuja (Thuja occidentalis L.) (48.7-51.5\%) [121-123], Dalmatian sage (Salvia officinalis L.) (13.1-48.5\%) [36], white wormwood (Artemisia herba-alba Asso) (25.7-36.8\%) [23,41,69], lanyana (Artemisia afra Jacq. ex Willd.) (22.5\%) [22], and common mugwort (Artemisia vulgaris L., camphor/thujone chemotype) (11.4\%) [49]. (R)- $\beta$-Thujone is found in wormwood ( $\beta$-thujone chemotype) (33.1-59.9\%) [23,41,69], tansy (Tanacetum vulgare L.) $(45.2 \%)$ [117], great mugwort (Artemisia arborescens (Vaill.) L.) (34.0\%) [39], wormwood ( $\beta$-thujone/(Z)epoxyocimene chemotype) (20.9-21.7\%), Dalmatian sage (3.9-19.1\%) [36], western red cedar (4.9-15.2\%) [68], génépi (10.4\%) [38], thuja (3.14-9.9\%) [121-123], white wormwood $(2.0-9.0 \%)[23,41,69]$, and lanyana (8.9\%) [22]. $\alpha$-Thujone is more neurotoxic than $\beta$-thujone. When taken orally, thujone can affect the CNS and cause convulsions which suggests that it can cross the blood-brain barrier [124,125]. Even at low doses, thujone can affect nervous tissue in rats [126]. Thujone diastereomers have been shown to inhibit human gammaaminobutyric acid type $\mathrm{A}\left(\mathrm{GABA}_{\mathrm{A}}\right)$ receptor currents $[127,128]$ which is the mechanism behind causing muscle spasms and convulsions $[129,130]$. Thujone has a NOAEL for convulsions of $5 \mathrm{mg} / \mathrm{kg}$ in female rats [131]. CYP2A6, followed by CYP3A4 and CYP2B6, metabolizes $\alpha$-thujone to 4 - and 7-hydroxythujone in humans [130]. $\alpha$-Thujone was found to inhibit CYP2A6 $\left(\mathrm{IC}_{50}=2.34 \mathrm{mg} / \mathrm{L}\right)$ and CYP2B6 $\left(\mathrm{IC}_{50}=2.66 \mathrm{mg} / \mathrm{L}\right)$ which could contribute to a lengthy and amplified $\alpha$-thujone toxicity [132]. Dalmatian sage EO causes convulsions [133]. Thuja, a thujone-rich EO, is abortifacient and contraceptive [122]. Ingestion of thuja oil may cause seizures, convulsions, hypotension, and gastroenteritis [121], and in severe cases, it can cause coma then death [123]. Tansy oil is neurotoxic and carries a risk of causing convulsions [117]. Treatment of female Swiss pregnant mice with methanolic extract of Artemisia herba-alba (intragastric gavage at 80 and $150 \mathrm{mg} / \mathrm{kg}$ ) in the entire period of gestation reduced fertility, altered the physical developments of the offspring, and delayed memory function and neuromotor reflex in the offspring [134]. The aqueous extract of Artemisia herba-alba (at $300 \mathrm{mg} / \mathrm{kg} /$ day) caused a decrease in fertility ratio of Sprague Dawley female rats [135]. The toxic effects in these studies were attributed to the flavonoid and thujone contents. Based on the available information, consumption of thujone-rich oils should be avoided in pregnancy. 


\section{Apiole-Rich Essential Oils}

For many years, parsley (Petroselinum crispum (Mill.) Fuss) and its concentrated preparations have been used in South America and Italy to induce abortion, which often ended in death due to severe post abortive vaginal bleeding [136]. The abortifacient effect is attributed to parsley apiole, a main component in most parsley leaf and seed oils. Parsley apiole and dill apiole are bicyclic phenylpropenoid ethers. Parsley apiole is found in parsley seed oil (11.3-67.5\%) [58] while dill apiole is found in Indian dill (Anethum sowa Roxb. ex Flem.) seed oil (20.7-52.5\%) [23] and parsley leaf oil (0.2-5.2\%) [22,57]. Parsley apiole poisoning causes severe neurotoxicity which presents a risk of abortion [137]. Signs of parsley apiole intoxication include fever, severe abdominal pain, vaginal bleeding, abortion, convulsions, vomiting, and diarrhea [138]. A single gavage dose of parsley apiole $(10 \mathrm{~mL} / \mathrm{kg})$ was sufficient to kill all experimental mice within 60 hours due to liver and kidney toxicity [139]. Doses of 5-14 g caused severe hemorrhage and induced abortion in pregnant rabbits [140]. Parsley leaf and seed oils are hepatotoxic, nephrotoxic, and may be abortifacient if taken orally. Topical application of parsley oils is also inadvisable during pregnancy [88]. Since there are no safety thresholds for parsley apiole in humans, internal and external use of parsley apiole-rich essential oils is not recommended in pregnancy due to the high risk of abortion. The structural similarity to parsley apiole suggests that dill apiole could carry the same toxicity and could be hazardous in pregnancy. Therefore, it is best to avoid apiole-rich oils (all routes) throughout pregnancy and breastfeeding [22].

\section{Camphor-Rich Essential Oils}

Camphor is a common component in many essential oils. It is a major component of Ho (Cinnamomum camphora (L.) J.Presl) leaf oil (camphor chemotype) (37.8-84.1\%) [40], feverfew (Tanacetum parthenium (L.) Sch.Bip.) oil (28.0-44.2\%) [37], and Spanish lavender (Lavandula stoechas L.) oil [64]. Upon consumption, camphor is absorbed immediately via the mucosa and can freely cross the placenta in pregnant women [98] and reach the fetal organs such as brain, liver, lungs, and kidneys [141]. At very high doses, camphor can cause hemorrhage due to severe damage to the placenta [142]. In female mice, camphor $(300 \mathrm{mg} / \mathrm{kg} /$ day for 20 days) increased the activities of hepatic CYP, glutathione $S$-transferase, and aryl hydrocarbon hydroxylase [143]. Camphor is very toxic to humans with a lethal dose of 5-20 g [144] and 50-550 mg/kg [98]. Camphor can cause damage to several organs including liver, kidney, and brain $[145,146]$. It can also cause convulsions [147] and induce seizures [148]. Signs of camphor poisoning include seizures, lack of coordination, respiratory depression, nausea, vomiting, and coma $[125,149,150]$. Despite being neurotoxic, hepatotoxic, and lethal in high doses, camphor is unexpectedly non-teratogenic and non-embryotoxic. In almost fatal doses, it can be reproductively toxic and abortifacient because the fetus lacks the necessary enzymes to metabolize it [151]. Camphor caused a dose-dependent maternal toxicity when given orally to pregnant rats $(0.216,0.464$, or $1 \mathrm{~g} / \mathrm{kg} /$ day on gestational days $6-17)$ and pregnant rabbits $(0.147,0.316$, or $0.681 \mathrm{~g} / \mathrm{kg} /$ day on gestational days 6-18) [152]. Yet, since camphor is believed to be more toxic to humans than animals, camphor-rich oils should be avoided in pregnancy and breastfeeding.

\section{Citral-Rich Essential Oils}

Citral, 3,7-dimethyl-2,6-octadien-1-al, is a mixture of two geometric isomers, geranial (citral a) and neral (citral b). Citral is a major component in a variety of essential oils including lemon myrtle (Backhousia citriodora F. Muell.) (<90.0\%) [42], East Indian lemongrass (Cymbopogon flexuosus (Nees ex Steud.) W. Watson) (83.0-90\%) [41,44,45], West Indian lemongrass (Cymbopogon citratus (DC.) Stapf) $(77.0-90 \%)$ [41,44,45], lemon-scented tea tree (Leptospermum petersonii F.M. Bailey) (77\%) [46], may chang (Litsea cubeba (Lour.) Pers.) (74-78\%) [41,48], lemon verbena (Aloysia triphylla L'Hérit) (68.0\%) [67], honey myrtle (Melaleuca teretifolia Endl.) (66.5\%) [22], Melissa (Melissa officinalis L.) (64.4\%) [37,48,153], lemon (Citrus limon (L.) Osbeck) leaf (50.0\%) [32], lemon basil (Ocimum $\times$ africanum Lour.) 
(42.2\%) [22], Australian lemon balm (Eucalyptus staigeriana F. v. Muell. ex F.M. Bailey) $(17.6 \%)$ [22], and lemon thyme (Thymus $\times$ citriodorus (Pers.) Schreb.) (16.3\%) [43]. Citral has a GRAS status and has been added as a flavoring and scenting agent to foods, cosmetics, and various household products (such as detergents, soaps, air fresheners, and insect repellents) to give a lemon or verbena scent. It is also an intermediate for ionone, methylionone, and vitamin A syntheses [154].

Citral is not mutagenic or carcinogenic [155]. However, it has shown some reproductive toxicity in animal studies. Citral reduced the fertility of female Wistar rats through decreasing the number of normal ovarian follicles [156]. Citral is a well-known retinoic acid synthesis inhibitor [157-160]. In epithelial tissues, citral has been shown to antagonize the activity of vitamin A and prevent the oxidation of retinol to retinoic acid [161]. In mouse epidermis, citral inhibited tissue morphogenesis and tumor production $[159,162]$. When tested on embryos of white Leghorn chicken, citral showed a dose-dependent teratogenic effect represented by inducing malformations and abnormal eye development [163-165]. Citral was reported to act via suppressing the activity of the enzyme ALDH1A1 responsible for retinoic acid synthesis, which in turn affects fetal development [166]. Citral (55 mM) partially inhibited the initiation of meiotic division in human fetal ovary tissues which relies partially on retinoic acid [166]. The oral NOAEL for citral-induced prenatal toxicity was set as $<60 \mathrm{mg} / \mathrm{kg} /$ day [167]. Orally administered citral (at 60, 125, 250, 500, and $1000 \mathrm{mg} / \mathrm{kg}$ on days 6 to 15 of pregnancy) produced signs of embryo-fetotoxicity (growth retardation, skeletal abnormalities, and spleen weight increase) and maternal toxicity (decreased maternal weight gain, increased resorptions, and impaired implantation) in pregnant Wistar rats [167]. Citral was teratogenic in studies with chick embryos [168,169]. The teratogenic effects of citral were also observed in Xenopus embryos treated with $60 \mathrm{mM}$ [170]. After exposure to $1.75 \mathrm{mM}$ of citral for $24 \mathrm{~h}$, tooth development was completely inhibited in $70 \%$ CD-1 Swiss mouse embryonic mandible explants while the addition of retinoic acid restored odontogenesis [171]. When injected intra-abdominally into pregnant BALB/c mice ( $>35 \mathrm{mmol} / \mathrm{g}$ on the 9 th gestational day), citral caused fetal cranial chondrogenesis and osteogenesis restrictions that diminished by adulthood [158]. However, when given to pregnant Wistar rats (by gavage at 125, 250,500, or $1000 \mathrm{mg} / \mathrm{kg}$ on gestational days 6-15), citral caused maternal toxicity, a dose-dependent increase in resorptions per implantation, and a slight teratogenicity [167]. The mechanism of action seems to involve competing with estrogen for estrogen receptors [172]. When applied directly to the rat's vagina, citral showed estrogenic effects and caused vaginal hyperplasia [172]. Inhalation of citral (for $6 \mathrm{hr} /$ day on gestation days 6-15 at 10 or 34 ppm as vapor, or $68 \mathrm{ppm}$ as an aerosol/vapor mixture) did not cause teratogenicity in Sprague-Dawley rats [168]. Due to their high citral content, Australian lemon balm, honey myrtle, lemon basil, lemon petitgrain, lemon myrtle, lemon thyme, lemongrass, lemon tea tree, May chang, Melissa, and lemon verbena EOs are teratogenic and their internal use should be restricted during pregnancy [153].

\section{9. $\beta$-Pulegone-Rich Essential Oils}

$\beta$-Pulegone ( $p$-menth-4(8)-en-3-one) is a monocyclic monoterpenoid ketone found in European pennyroyal (Mentha pulegium L.) (67.6-86.7\%) [23], North American pennyroyal (Hedeoma pulegioides (L.) Pers.) (61.3-82.3\%) [23], lesser calamint (Calamintha nepeta (L.) Savi) (17.6-76.1\%) [47], buchu (Agathosma betulina (P.J. Bergius) Pillans) (pulegone chemotype) $(31.6-73.2 \%)$ [29,30], Turkish pennyroyal (Micromeria fruticosa (L.) Druce) (66.7\%) [23], and buchu (Agathosma crenulata L.) (diosphenol chemotype) (0.6-4.5\%) [29]. Following oral consumption, pulegone is metabolized into at least 14 metabolites including menthofuran and 8-pulegone aldehyde which are more toxic than pulegone itself [173-175]. Pulegone metabolites are the ones responsible for the toxicity since menthofuran is hepatotoxic and $p$-cresol is a toxin and glutathione-depleting agent $[176,177]$. $\gamma$-Ketoenal, a reactive metabolite of pulegone, causes hepatic injury via covalently binding to cellular proteins in the liver [176,177]. Treatment with cytochrome P-450 inhibitors (SKF-525A, metyrapone, piperonyl butoxide, and carbon disulfide) blocked pulegone hepatotoxicity, indicating the 
involvement of cytochrome P-450 in pulegone metabolism [175,178]. Unlike menthofuran, $(1 R)-(+)-\beta$-pulegone was reported to deplete hepatic glutathione in in vitro experiments, and upon i.p. injection into rats (at $150 \mathrm{mg} / \mathrm{kg}$ ) or mice (at $300 \mathrm{mg} / \mathrm{kg}$ ) $[173,179]$. In rats, $(1 R)-(+)-\beta$-pulegone (i.p., at $300 \mathrm{mg} / \mathrm{kg}$ ) caused severe damage to the endoplasmic reticulum that led to cell death [180]. (1R)-(+)- $\beta$-Pulegone treatment was found to destroy hepatic cytochrome P-450 enzymes [178,180-182].

For a long time, pennyroyal has been used as an abortifacient even with its potentially deadly hepatotoxic effects [183]. Pennyroyal oil is hepatotoxic and neurotoxic due to the high content of (6R)-(+)-menthofuran and (1R)-(+)- $\beta$-pulegone [179]. Both Mentha pulegium oil and pulegone prevented rat uterine muscle contraction [8]. Pennyroyal intoxication causes severe liver damage, internal hemorrhage, and pulmonary edema [184,185]. Intraperitoneal administration of both pennyroyal oil and pulegone showed similar effects in mice [179]. Since there is no significant medicinal benefit from using $\beta$-pulegone-rich oils and due to their hepatotoxicity and the potential of causing abortion, it is best to avoid them in pregnancy and breastfeeding [186].

\section{Costunolide and Dehydrocostus Lactone-Rich Essential Oils}

Costunolide or costus lactone is a bicyclic sesquiterpenoid polyalkene lactone found in costus (Saussurea costus (Falc.) Lipsch.) oil (11.0\%) [35]. Costunolide is classified as a skin sensitizer (allergen) [187]. Costunolide (i.p., at $100 \mathrm{mg} / \mathrm{kg} /$ day) showed anti-angiogenic activity through decreasing vascular endothelial growth factor (VEGF)-induced neovascularization in mice. It also suppressed umbilical vein endothelial cell proliferation in vitro $\left(\mathrm{IC}_{50}=3.4 \mathrm{mM}\right)$ [188]. Dehydrocostus lactone $(\mathrm{DHC})$ is a sesquiterpenoid lactone found in costus $(6.0 \%)$. Similar to costus lactone, dehydrocostus lactone is a skin sensitizer [187]. Dehydrocostus lactone exerts its anti-angiogenic action via causing $\mathrm{G}_{0} / \mathrm{G}_{1}$ cell cycle arrest

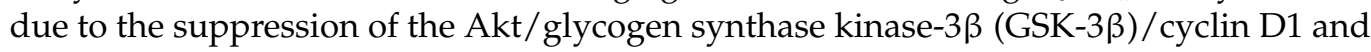
mTOR signaling pathway [189]. Dehydrocostus lactone inhibited angiogenesis in vitro and in mice in a dose-dependent manner [189]. Costus oil is a good example for costunolide and dehydrocostus lactone-rich essential oils [35]. Costus EO is fetotoxic due to its costunolide and dehydrocostus lactone content. Due to the strong anti-angiogenic activity of costunolide and dehydrocostus lactones $[188,189]$ and because of the possible link between anti-angiogenic activity and reproductive toxicity $[13,14]$, it is best to avoid the oil (by any route) during pregnancy and lactation.

\section{Thymoquinone-Rich Essential Oils}

Thymoquinone is a bicyclic benzenoid ketone found in black seed (Nigella sativa L.) oil (26.8-54.8\%) [27]. It showed reproductive toxicity with an i.p. NOAEL of $15 \mathrm{mg} / \mathrm{kg}$. When administered daily to rats (i.p., at $8 \mathrm{mg} / \mathrm{kg}$ ), thymoquinone killed most of the animals within a week and the surviving animals had severe peritonitis [190]. It suppressed VEGF-induced angiogenesis in the matrigel plug assay. Subcutaneous administration of thymoquinone to mice (at $6 \mathrm{mg} / \mathrm{kg}$ for 15 days) abolished angiogenesis in prostate cancer tumors [191]. Administration of a single dose of thymoquinone (i.p., at 35 or $50 \mathrm{mg} / \mathrm{kg}$ on gestational days 11 or 14) to pregnant rats caused a dose-dependent fetal resorption and maternal toxicity [192]. Thymoquinone (by gavage at $10 \mathrm{mg} / \mathrm{kg} /$ day on gestational days 1-19) reduced malondialdehyde formation and increased hepatic glutathione in mice with induced gestational diabetes [193]. Due to its strong anti-angiogenic activity [191] and reproductive toxicity, thymoquinone is mostly hazardous in pregnancy. Black seed oil may be fetotoxic because of its high thymoquinone content [191]; therefore, its consumption during pregnancy and breastfeeding should be avoided.

\section{2. $\beta$-Elemene- and/or $\beta$-Eudesmol-Rich Essential Oils}

$\beta$-Elemene, a monocyclic sesquiterpenoid polyalkene, is one of the major components in atractylis (Atractylodes lancea (Thunb.) DC) EO (18.0\%) [22], myrrh (Commiphora myrrha (Nees) Engl.) EO (8.7\%) [50], and katrafay (Cedrelopsis grevei Baill. and Courchet) EO 
(3.0-6.0\%) [22]. It is known for its strong anti-angiogenic effects through inhibiting VEGF expression and repressing VEGF-dependent tumor angiogenesis [194-199]. Myrrh oil may be fetotoxic based on its $\beta$-elemene [174] and furanodiene [200] content and therefore, should be avoided during pregnancy.

$\beta$-Eudesmol, a bicyclic sesquiterpenoid alkene alcohol, is a major component in atractylis (Atractylodes lancea (Thunb.) DC) (26.0\%) [22], araucaria (Neocallitropsis pancheri (Carrière) de Laub.) (25.9\%) [26], blue cypress (Callitris intratropica R.T. Baker and H.B. Sm.) (14.4\%) [28], European valerian (Valeriana officinalis L.) (0-8.3\%), amyris (Amyris balsamifera L.) (3.2-7.9\%), blue tansy (Tanacetum annuum L.) (3.5-6.7\%) [117], hinoki leaf (Chamaecyparis obtusa (Siebold and Zucc.) Endl.) (6.5\%), Eucalyptus smithii (6.3\%), emerald cypress (Callitris columellaris F.Muell.) (5.7\%), and vetiver (Vetiveria zizanioides (L.) Nash) $(0-5.2 \%)$ [22]. $\beta$-Eudesmol is a known anti-angiogenic agent both in vitro and in vivo via blocking of the extracellular signal-regulated kinase (ERK) signaling pathway [201]. $\beta$ Eudesmol showed significant in vitro antiplatelet aggregation activity and best be avoided in childbirth [22]. Based on their $\beta$-eudesmol content, atractylis, araucaria, and blue cypress oils may be fetotoxic and may inhibit blood clotting; they should be avoided during pregnancy and lactation.

\section{Other Essential Oils}

(E)-Cinnamaldehyde-rich oils, such as cassia (Cinnamomum cassia (L.) J. Presl) [23] and cinnamon (Cinnamomum verum J. Presl) bark oil [23,24], carry a risk of embryotoxicity and should be avoided during pregnancy and breastfeeding. Cinnamon bark oil has GRAS status, yet it has been shown to lower the number of nuclei and affect the distribution of embryos in pregnant mice (orally, at $375 \mathrm{mg} / \mathrm{kg}$ for 2 weeks) [202].

Methyleugenol, a major component in chaste tree (Vitex agnus-castus L.) oil, can cause reproductive hormone modulation [33,34]. Moreover, methyleugenol has demonstrated hepatocarcinogenicity in rodents via inducing unscheduled DNA synthesis and forming DNA and protein adducts [89]. It is inadvisable to use methyleugenol-containing oils during pregnancy and breastfeeding. Vitex agnus-castus is known to ameliorate premenstrual syndrome (PMS), menstrual cycle irregularity, dysfunctional uterine bleeding, and cyclical breast discomfort [203]. Chaste tree fruit and leaf EOs can alleviate menopausal symptoms [204-206]. However, the extract of chaste tree showed dopaminergic activity both in vitro and in vivo via lowering serum estrogen and increasing progesterone levels [204]. Chaste tree fruit and leaf EOs can stimulate dopamine D2 receptors which inhibit prolactin release and normalize the menstrual cycle. Likewise, hyssop (Hyssopus officinalis L.) oil (pinocamphone chemotype) is neurotoxic and potentially carcinogenic based on its methyleugenol content [37,41]. Ingestion of hyssop oil might cause epileptiform convulsions [207] and seizures due to its high pinocamphone content [121]. Hyssop oil and cis- and trans-3-pinanones are $\mathrm{GABA}_{\mathrm{A}}$ receptor antagonists [208]. Nutmeg (Myristica fragrans Houtt.) oil is potentially carcinogenic because of its safrole and methyleugenol content $[23,51,89]$.

Some oil chemotypes like the estragole chemotype of basil (Ocimum basilicum L.) oil [23] are toxic based on their estragole content which, in high concentrations, is carcinogenic and should be restricted during pregnancy and lactation [88,89]. Dalmatian sage oil carries a combined risk from its camphor and thujone contents which makes it neurotoxic and embryotoxic [36]. The oil should not be taken orally and its consumption is contraindicated in pregnancy and breastfeeding [88]. Ingestion of dalmatian sage oil can cause convulsions, seizure, coma, and may lead to death [209,210]. Dalmatian sage oil $(0.25 \%, 375 \mathrm{mg} / \mathrm{kg}$ for 2 weeks) negatively influenced the distribution of embryos according to nucleus number when fed to pregnant mice [202]. Since the risks of dalmatian sage oil outweigh its benefits, it is best to avoid using it. Hibawood (Thujopsis dolabrata (L.f.) Siebold and Zucc.) EO may present a reproductive toxicity because of its $\beta$-thujaplicin content [22]. In rats, orally delivered $\beta$-thujaplicin caused fetal malformations at $135 \mathrm{mg} / \mathrm{kg}$ 
and a decrease in fetal weight at $45 \mathrm{mg} / \mathrm{kg}$ [211]. Nasturtium (Tropaeolum majus L.) flower absolute carries a moderate toxicity because of its benzyl cyanide content [22].

In some cases, the hazardous components in the oil have not been identified yet. For instance, carrot (Daucus carota L.) seed oil has GRAS status; however, it may interfere with gestation and should be avoided altogether during pregnancy and breastfeeding. It is worth mentioning that the wild carrot plant is reputed as a contraceptive agent. Dong and colleagues have reported that carrot seed oil caused antigestational effects in rats and mice [212]. Subcutaneous injection of carrot seed EO $(2.5-5 \mathrm{~mL} / \mathrm{kg})$ to female rats and mice inhibited implantation and prevented progesterone synthesis [213]. Another example is oregano (Origanum vulgare L.) oil [53-56]. Although oregano oil has a GRAS status, it is embryotoxic. Orally delivered Origanum vulgare EO to pregnant mice (about $150 \mathrm{mg} / \mathrm{kg}$ for two weeks) caused an increase in the rate of embryonic cell death [202]. Additionally, zedoary (Curcuma zedoaria (Christm.) Roscoe) oil has a GRAS status but its consumption can interfere with gestation and can induce abortion [22]. There was obvious embryotoxicity for zedoary EO ex vivo and reproductive toxicity in animal and developmental experiments [14,174]. In addition, the oil was anti-angiogenic in mice [174], suggesting a strong link between its anti-angiogenic and embryotoxic effects [14]. Chinese zedoary EO (i.p., $300 \mathrm{mg} / \mathrm{kg}$ ) prevented implantation in a dose-dependent manner in female rats on gestational days $7-9$ and prevented about $77 \%$ of pregnancies. When administered intra-vaginally to female rabbits at 60 or $400 \mathrm{mg} / \mathrm{kg} /$ day on gestational days $5-9$ and $2-4$, a steam-distilled zedoary EO prevented $16 \%$ and $100 \%$ of pregnancies, respectively [22]. Aqueous extracts of C. zedoaria rhizome (10 g/ kg/day for 20 days) demonstrated reproductive toxicity in pregnant mice [214]. The embryotoxic effect of zedoary EO was attributed to its sesquiterpenoids, which can block VEGF-mediated angiogenesis [14]. Nevertheless, there is no direct evidence to link any of the oil components to its antifertility effect. Moreover, zedoary rhizome decoctions and ethanol extracts also have antifertility effects [215].

Rue (Ruta graveolens L.) oil may be abortifacient and should be strictly prohibited in pregnancy and breastfeeding. In South America, Ruta graveolens ingestion caused abortion [136]. Ingestion of rue aqueous extract was abortifacient, and inhibited implantation in rats at $1 \mathrm{~mL} / \mathrm{kg}$ [9]. Rue chloroform extract showed antifertility effects due to the presence of chalepensin [9]. Pilocarpine, a compound not present in rue oil, has been suggested as the abortifacient agent [216]. Very little information was found about the toxicity and safety of rue oil. Until further data are available, rue oil should be avoided altogether during pregnancy.

In summary, if essential oil constituents are present in the mother's circulation, they are expected to reach the fetus and exert some toxic effects. Due to the lack of clinical evidence on reproductive toxicity in humans, it is best to avoid or restrict the use of potentially dangerous essential oil constituents such as anethole, apiole, citral, camphor, thymoquinone, trans-sabinyl acetate, methyl salicylate, thujone, pulegone, $\beta$-elemene, $\beta$-eudesmol, and costus lactone.

Author Contributions: Writing-Original Draft Preparation, N.S.D.; Writing-Review and Editing, N.S.D. and W.N.S. Both authors have read and agreed to the published version of the manuscript.

Funding: This work was carried out as part of the activities of the Aromatic Plant Research Center. (APRC, https:/ / aromaticplant.org/) (accessed on 29 January 2021).

Conflicts of Interest: The authors declare no conflict of interest. 


$\begin{array}{ll}\text { Abbreviations } \\ \text { EO } & \begin{array}{l}\text { essential oil } \\ \text { central nervous system }\end{array} \\ \text { GSK-3 } \beta & \begin{array}{l}\text { glycogen synthase kinase-3 } \beta \\ \text { subcutaneous }\end{array} \\ \text { sc } & \text { premenstrual syndrome } \\ \text { PMS } & \text { generally recognized as safe } \\ \text { GRAS } & \text { intraperitoneal } \\ \text { i.p. } & \text { median inhibitory concentration } \\ \text { IC } 50 & \text { no observed adverse effect level } \\ \text { NOAEL } & \text { parts per million } \\ \text { ppm } & \text { vascular endothelial growth factor } \\ \text { VEGF } & \text { gamma-aminobutyric acid type A }\end{array}$

\section{References}

1. Howes, M.-J.R.; Houghton, P.J.; Barlow, D.J.; Pocock, V.J.; Milligan, S.R. Assessment of estrogenic activity in some common essential oil constituents. J. Pharm. Pharmacol. 2002, 54, 1521-1528. [CrossRef]

2. Ososki, A.L.; Kennelly, E.J. Phytoestrogens: A review of the present state of research. Phyther. Res. 2003, 17, 845-869. [CrossRef] [PubMed]

3. Azaizeh, H.; Saad, B.; Cooper, E.; Said, O. Traditional Arabic and Islamic medicine, a re-emerging health aid. Evidence-Based Complement. Altern. Med. 2010, 7, 419-424. [CrossRef]

4. Al-Ramahi, R.; Jaradat, N.; Adawi, D. Use of herbal medicines during pregnancy in a group of Palestinian women. J. Ethnopharmacol. 2013, 150, 79-84. [CrossRef]

5. Mbemya, G.T.; Vieira, L.A.; Canafistula, F.G.; Pessoa, O.D.L.; Rodrigues, A.P.R. Reports on in vivo and in vitro contribution of medicinal plants to improve the female reproductive function. Reprodução Clim. 2017, 32, 109-119. [CrossRef]

6. Ali-Shtayeh, M.S.; Jamous, R.M.; Jamous, R.M. Plants used during pregnancy, childbirth, postpartum and infant healthcare in Palestine. Complement. Ther. Clin. Pract. 2015, 21, 84-93. [CrossRef] [PubMed]

7. Macht, D.I. The action of so-called emmenagogue oils on the isolated uterine strip. J. Pharm. Exp. Ther. 1913, 4, 547-553.

8. Soares, M.C.M.S.; Damiani, C.E.N.; Moreira, C.M.; Stefanon, I.; Vassallo, D.V. Eucalyptol, an essential oil, reduces contractile activity in rat cardiac muscle. Braz. J. Med. Biol. Res. 2005, 38, 453-461. [CrossRef]

9. Kong, Y.C.; Lau, C.P.; Wat, K.H.; Ng, K.H.; But, P.P.H.; Cheng, K.F.; Waterman, P.G. Antifertility Principle of Ruta graveolens. Planta Med. 1989, 55, 176-178. [CrossRef]

10. Datnow, M.M. An experimental investigation concerning toxic abortion produced by chemical agents. J. Obs. Gynaecol. 1928, 35, 693-724. [CrossRef]

11. Diel, P.; Smolnikar, K.; Michna, H. In vitro test systems for the evaluation of the estrogenic activity of natural products. Planta Med. 1999, 65, 197-203. [CrossRef] [PubMed]

12. Timbrell, J.A. Principles of Biochemical Toxicology, 3rd ed.; Taylor \& Francis: Padstow, UK, 2000.

13. Chaiworapongsa, T.; Romero, R.; Kusanovic, J.P.; Savasan, Z.A.; Kim, S.K.; Mazaki-Tovi, S.; Vaisbuch, E.; Ogge, G.; Madan, I.; Dong, Z.; et al. Unexplained Fetal Death is Associated with Increased Concentrations of Anti-Angiogenic Factors in Amniotic Fluid. J. Matern. Fetal Neonatal. Med. 2010, 23, 794-805. [CrossRef]

14. Zhou, L.; Zhang, K.; Li, J.; Cui, X.; Wang, A.; Huang, S.; Zheng, S.; Lu, Y.; Chen, W. Inhibition of vascular endothelial growth factor-mediated angiogenesis involved in reproductive toxicity induced by sesquiterpenoids of Curcuma zedoaria in rats. Reprod. Toxicol. 2013, 37, 62-69. [CrossRef]

15. Ogata, A.; Ando, H.; Kubo, Y.; Nagasawa, A.; Ogawa, H.; Yasuda, K.; Aoki, N. Teratogenicity of Thujaplicin in ICR Mice. Food Chem. Toxicol. 1999, 37, 1097-1104. [CrossRef]

16. Reynolds, J.E.F. Martindale: The Extra Pharmacopoeia; The Pharmaceutical Press: London, UK, 1993.

17. Maickel, R.P.; Snodgrass, W.R. Physicochemical factors in maternal-fetal distribution of drugs. Toxicol. Appl. Pharmacol. 1973, 26, 218-230. [CrossRef]

18. Forschmidt, P.; Liban, E.; Abramovici, A. Teratogenic activity of flavour additives. Teratology 1979, 19, 26A.

19. Jori, A.; Briatico, G. Effect of eucalyptol on microsomal enzyme activity of foetal and newborn rats. Biochem. pharmacol. 1973, 22, 543-544. [CrossRef]

20. Hausner, H.; Bredie, W.L.; Molgaard, C.; Petersen, M.A.; Moller, P. Differential transfer of dietary flavour compounds into human breast milk. Physiol. Behav. 2008, 95, 118-124. [CrossRef]

21. Chhabra, S.K.; Rao, A.R. Postnatal modulation of xenobiotic metabolizing enzymes in liver of mouse pups following translactational exposure to sandalwood oil. Nutr. Res. 1993, 13, 1191-1202. [CrossRef]

22. Tisserand, R.; Young, R. Essential Oil Safety, 2nd. ed.; Elsevier: Amsterdam, The Netherlands, 2014.

23. Lawrence, B.M. Essential Oils 1988-1991; Allured Publishing: Wheaton, IL, USA, 1995. 
24. Kubeczka, K.-H.; Formacek, V. Essential Oils Analysis by Capillary Gas Chromatography and Carbon-13NMR Spectroscopy, 2nd ed.; J. Wiley \& Sons: New York, NY, USA, 2002.

25. Brophy, J.J.; Goldsack, R.J.; Fookes, C.J.R.; Forster, P.I. Leaf Oils of the Genus Backhousia (Myrtaceae). J. Essent. Oil Res. 1995, 7, 237-254. [CrossRef]

26. Raharivelomanana, P.; Cambon, A.; Azzaro, M.; Bianchini, J.P.; Claude-Lafontaine, A.; George, G. Volatile constituents of Neocallitropsis pancheri (Carriere) de Laubenfels heartwood extracts (Cupressaceae). J. Essent. Oil Res. 1993, 5, 587-595. [CrossRef]

27. Mozaffari, F.S.; Ghorbanli, M.; Babai, A.; Sepehr, M.F. The Effect of Water Stress on the Seed Oil of Nigella sativa L. J. Essent. Oil Res. 2000, 12, 36-38. [CrossRef]

28. Doimo, L. Azulenes, Costols and $\gamma$-Lactones from Cypress-Pines (Callitris columellaris, C. glaucophylla and C. intratropica) Distilled Oils and Methanol Extracts. J. Essent. Oil Res. 2001, 13, 25-29. [CrossRef]

29. Collins, N.F.; Graven, E.H.; Van Beek, T.A.; Lelyveld, G.P. Chemotaxonomy of Commercial Buchu Species (Agathosma betulina and A. crenulata). J. Essent. Oil Res. 1996, 8, 229-235. [CrossRef]

30. Posthumus, M.A.; van Beek, T.A.; Collins, N.F.; Graven, E.H. Chemical Composition of the Essential Oils of Agathosma betulina, A. crenulata and an A. betulina x crenulata Hybrid (Buchu). J. Essent. Oil Res. 1996, 8, 223-228. [CrossRef]

31. Mazzoni, V.; Tomi, F.; Casanova, J. A daucane-type sesquiterpene from Daucus carota seed oil. Flavor Fragr. J. 1999, 14, $268-272$. [CrossRef]

32. Lawrence, B.M. Brogress in essential oils. Perfum. Flavorist 1995, 20, 48-49.

33. Senatore, F.; Della Porta, G.; Reverchon, E. Constituents of Vitex agnus-castus L. Essential Oil. Flavor Fragr. J. 1996, 11, 179-182. [CrossRef]

34. Sørensen, J.M.; Katsiotis, S.T. Parameters influencing the yield and composition of the essential oil from Cretan Vitex agnus-castus fruits. Planta Med. 2000, 66, 245-250. [CrossRef]

35. Mahindru, S.N. Indian Plant Perfumes; Metropolitan: New Delhi, India, 1992.

36. Lawrence, B.M. Progress in essential oils. Perfum. Flavorist 1998, 23, 50.

37. Lawrence, B.M. Progress in essential oils. Perfum. Flavorist 1999, 24, 56-59.

38. Mucciarelli, M.; Caramiello, R.; Maffei, M.; Chialva, F. Essential oils from some Artemisia species growing spontaneously in North-West Italy. Flavor Fragr. J. 1995, 10, 25-32. [CrossRef]

39. Tucker, A.O.; Maciarello, M.J.; Sturtz, G. The essential oils of Artemisia 'Powis Castle' and its Putative Parents, A. absinthium and A. arborescens. J. Essent. Oil Res. 1993, 5, 239-242. [CrossRef]

40. Pélissier, Y.; Marion, C.; Prunac, S.; Bessière, J.-M. Volatile Components of Leaves, Stems and Bark of Cinnamonum camphora Nees et Ebermaier. J. Essent. Oil Res. 1995, 7, 313-315.

41. Lawrence, B.M. Essential Oils 1981-1987; Allured Publishing: Wheaton, IL, USA, 1989.

42. Southwell, I.A.; Russell, M.; Smith, R.L.; Archer, D.W. Backhousia citriodora F. Muell. (Myrtaceae), A Superior Source of Citral. J. Essent. Oil Res. 2000, 12, 735-741. [CrossRef]

43. Horváth, G.; Szabó, L.G.; Héthelyi, É.; Lemberkovics, É. Essential Oil Composition of Three Cultivated Thymus Chemotypes from Hungary. J. Essent. Oil Res. 2006, 18, 315-317.

44. Lawrence, B.M. Progress in essential oils: Ocimum gratissimum oil, cinnamon oil, tarragon oil, and palmarosa oil. Perfum. Flavorist 2002, 27, 42-52.

45. Chagonda, L.S.; Makanda, C.; Chalchat, J.C. Essential oils of cultivated Cymbopogon winterianus (Jowitt) and of C. citratus (DC) (Stapf) from Zimbabwe. J. Essent. Oil Res. 2000, 12, 478-480. [CrossRef]

46. Brophy, J.J.; Goldsack, R.J.; Bean, A.R.; Forster, P.I.; Lepsch, B.J. Leaf essential oils of the genus Leptospermum (Myrtaceae) in eastern Australia, Part 6. Leptospermum polygalifolium and allies. Flav. Fragr. J. 2000, 15, 271-277. [CrossRef]

47. Ristorcelli, D.; Tomi, F.; Casanova, J. Essential Oils of Calamintha nepeta subsp. nepeta and subsp. glandulosa from Corsica (France). J. Essent. Oil Res. 1996, 8, 363-366. [CrossRef]

48. Lawrence, B.M. Progress in essential oils. Perfum. Flavorist 1996, 21, 59-60.

49. Haider, F.; Dwivedi, P.D.; Naqvi, A.A.; Bagchi, G.D. Essential oil composition of Artemisia vulgaris harvested at different growth periods under Indo-Gangetic plain conditions. J. Essent. Oil Res. 2003, 15, 376-378. [CrossRef]

50. Dekebo, A.; Dagne, E.; Sterner, O. Furanosesquiterpenes from Commiphora sphaerocarpa and related adulterants of true myrrh. Fitoterapia 2002, 73, 48-55. [CrossRef]

51. Simpson, G.I.; Jackson, Y.A. Comparison of the chemical composition of East Indian, Jamaican and other West Indian essential oils of Myristica fragrans Houtt. J. Essent. Oil Res. 2002, 14, 6-9. [CrossRef]

52. Kaiser, R. New Volatile Constituents of the Flower Concrete of Michelia champaca L. J. Essent. Oil Res. 1991, 3, 129-146. [CrossRef]

53. Baser, K.H.C.; Özek, T.; Kürkçüoglu, M.; Tümen, G. The Essential Oil of Origanum vulgare subsp. hirtum of Turkish Origin. J. Essent. Oil Res. 1994, 6, 31-36. [CrossRef]

54. Karpouhtsis, I.; Pardali, E.; Feggou, E.; Kokkini, S.; Scouras, Z.G.; Mavragani-Tsipidou, P. Insecticidal and genotoxic activities of Oregano essential oils. J. Agric. Food Chem. 1998, 46, 1111-1115. [CrossRef]

55. Baser, K.H.C.; Özek, T.; Tümen, G.; Sezik, E. Composition of the Essential Oils of Turkish Origanum Species with Commercial Importance. J. Essent. Oil Res. 1993, 5, 619-623. [CrossRef] 
56. Lagouri, V.; Blekas, G.; Tsimidou, M.; Kokkini, S.; Boskou, D. Composition and antioxidant activity of essential oils from Oregano plants grown wild in Greece. Zeitschrift für Leb. Forsch. 1993, 197, 20-23. [CrossRef]

57. Lawrence, B.M. Progress in essential oils. Perfum. Flavorist 2001, 26, 38.

58. Lamarti, A.; Badoc, A.; Bouriquet, R. A Chemotaxonomic Evaluation of Petroselinum crispum (Mill.) A. W. Hill (Parsley) Marketed in France. J. Essent. Oil Res. 1991, 3, 425-433. [CrossRef]

59. Fournier, G.; Paris, M.; Dumitresco, S.M.; Pages, N.; Boudene, C. Contribution to the study of Plectranthus fruticosus leaf essential oil. Planta Med. 1986, 6, 486-488. [CrossRef] [PubMed]

60. De Feo, V.; De Simone, F.; Senatore, F. Potential allelochemicals from the essential oil of Ruta graveolens. Phytochemistry 2002, 61, 573-578. [CrossRef]

61. SCCP Opinion on furocoumarins in cosmetic products. Scientific committee on consumer products. SCCP/0942/05. 2005. Available online: https:/ / ec.europa.eu/health/ph_risk/committees/04_sccp/docs/sccp_o_036.pdf (accessed on 29 January 2021).

62. Yatagai, M.; Sato, T.; Yakahashi, T. Terpenes of leaf oils from Cupressaceae. Biochem. Syst. Ecol. 1985, 13, 377-385. [CrossRef]

63. Mathela, C.S.; Kharkwal, H.; Shah, G.C. Essential Oil Composition of Some Himalayan Artemisia Species. J. Essent Oil Res. 1994, 6, 345-348. [CrossRef]

64. Ristorcelli, D.; Tomi, F.; Casanova, J. 13C-NMR as a tool for identification and enantiomeric differentiation of major terpenes exemplified by the essential oil of Lavandula stoechas L. ssp. stoechas. Flavor Fragr. J. 1998, 13, 154-158. [CrossRef]

65. Usano-Alemany, J.; Herraiz-Peñalver, D.; Cuadrado, J.; Díaz, S.; Santa-Cruz, M.; Palá-Paúl, J. Seasonal Variation of the Essential Oils of Salvia lavandulifolia: Antibacterial Activity. J. Essent. Oil Bear. Plants 2012, 15, 195-203. [CrossRef]

66. Singh, G.; Singh, O.P.; Maurya, S. Chemical and biocidal investigations on essential oils of some Indian curcuma species. Prog. Cryst. Growth Charact. Mater. 2002, 45, 75-81. [CrossRef]

67. Santos-Gomes, P.C.; Fernandes-Ferreira, M.; Vicente, A.M.S. Composition of the Essential Oils from Flowers and Leaves of Vervain (Aloysia triphylla (L’Herit.) Britton) Grown in Portugal. J. Essent. Oil Res. 2005, 17, 73-78. [CrossRef]

68. Von Rudloff, E.; Lapp, M.S.; Yeh, F.C. Chemosystematic study of Thuja plicata: Multivariate analysis of leaf oil terpene composition. Biochem. Syst. Ecol. 1988, 16, 119-125. [CrossRef]

69. Ghanmi, M.; Satrani, B.; Aafi, A.; Isamili, M.R.; Houti, H.; El Monfalouti, H.; Benchakroun, K.H.; Aberchane, M.; Harki, L.; Boukir, A. Effect of harvest date on yield, chemical composition and bioactivity of essential oils of sagebrush (Artemisia herbaalba) in the region of Guercif (eastern Morocco). Phytothérapie 2010, 8, 295-301. [CrossRef]

70. Angel, G.R.; Menon, N.; Vimala, B.; Nambisan, B. Essential oil composition of eight starchy Curcuma species. Ind. Crops Prod. 2014, 60, 233-238. [CrossRef]

71. Singh, P.; Singh, S.; Kapoor, I.P.S.; Singh, G.; Isidorov, V.; Szczepaniak, L. Chemical composition and antioxidant activities of essential oil and oleoresins from Curcuma zedoaria rhizomes, part-74. Food Biosci. 2013, 3, 42-48. [CrossRef]

72. Purkayastha, J.; Nath, S.C.; Klinkby, N. Essential oil of the rhizome of Curcuma zedoaria (Christm.) Rosc. Native to northeast India. J. Essent. Oil Res. 2006, 18, 154-155. [CrossRef]

73. Herath, H.M.I.C.; Wiyasiriwardene, T.D.C.M.K.; Premakumara, G.A.S. Comparative GC-MS analysis of all Curcuma species grown in Sri Lanka by multivariate test. Ruhunu J. Sci. 2017, 8, 1-9. [CrossRef]

74. Boissier, J.R.; Simon, P.; Le Bourhis, B. Experimental psychotropic action of cis and trans isomers of anethol. Therapie 1967, 22, 309-323. [PubMed]

75. Ritter, A.M.V.; Domiciano, T.P.; Verri, W.A.; Zarpelon, A.C.; Da Silva, L.G.; Barbosa, C.P.; Natali, M.R.M.; Cuman, R.K.N.; Bersani-Amado, C.A. Antihypernociceptive activity of anethole in experimental inflammatory pain. Inflammopharmacology 2013, 21, 187-197. [CrossRef] [PubMed]

76. Shahbazian, S.; Akbarzadeh, A.; Torabi, S.; Omidi, M. Anti-cancer activity of pegylated liposomal trans-anethole on breast cancer cell lines MCF-7 and T47D. Biotechnol. Lett. 2015, 37, 1355-1359. [CrossRef] [PubMed]

77. Domiciano, T.P.; Dalalio, M.M.H.D.O.; Silva, E.L.; Ritter, A.M.V.; Estevão-Silva, C.F.; Ramos, F.S.; Caparroz-Assef, S.M.; Cuman, R.K.N.; Bersani-Amado, C.A. Inhibitory effect of anethole in nonimmune acute inflammation. Naunyn. Schmiedebergs. Arch. Pharmacol. 2013, 386, 331-338. [CrossRef]

78. Albert-Puleo, M. Fennel and anise as estrogenic agents. J. Ethnopharmacol. 1980, 2, 337-344. [CrossRef]

79. Türkyilmaz, Z.; Karabulut, R.; Sönmez, K.; Başaklar, K.A. A striking and frequent cause of premature thelarche in children: Foeniculum vulgare. J. Pediatr. Surg. 2008, 43, 2109-2111.

80. Dhar, S.K. Anti-fertility activity and hormonal profile of trans-anethole in rats. Indian J. Physiol. Pharmacol. 1995, 39, 63-67. [PubMed]

81. Newberne, P.M.; Carlton, W.W.; Brown, W.R. Histopathological evaluation of proliferative liver lesions in rats fed trans-anethole in chronic studies. Food Chem. Toxicol. 1989, 27, 21-26. [CrossRef]

82. Kim, S.G.; Liem, A.; Stewart, B.C.; Miller, J.A. New studies on trans-anethole oxide and trans-asarone oxide. Carcinogenesis 1999, 20, 1303-1307. [CrossRef] [PubMed]

83. Marshall, A.D.; Caldwell, J. Influence of modulators of epoxide metabolism on the cytotoxicity of trans-anethole in freshly isolated rat hepatocytes. Food Chem. Toxicol. 1992, 30, 467-473. [CrossRef]

84. Ostad, S.N.; Khakinegad, B.; Sabzevari, O. Evaluation of the teratogenicity of fennel essential oil (FEO) on the rat embryo limb buds culture. Toxicol. In Vitro 2004, 18, 623-627. [CrossRef] 
85. Bilia, A.R.; Flamini, G.; Taglioli, V.; Morelli, I.; Vincieri, F.F. GC-MS analysis of essential oil of some commercial Fennel teas. Food Chem. 2002, 76, 307-310. [CrossRef]

86. Ostad, S.N.; Soodi, M.; Shariffzadeh, M.; Khorshidi, N.; Marzban, H. The effect of fennel essential oil on uterine contraction as a model for dysmenorrhea, pharmacology and toxicology study. J. Ethnopharmacol. 2001, 76, 299-304. [CrossRef]

87. Blumenthal, M.; Goldberg, A.; Brinckmann, J. Herbal Medicine: Expanded Commission E Monographs; Integrative Medicine Communications: Boston, MA, USA, 2000.

88. Blumenthal, M.; Busse, W.R.; Goldberg, A.; Gruenwald, J.; Hall, T.; Riggins, C.W.; Rister, R.S. The Complete German Commission E Monographs. Therapeutic Guide to Herbal Medicines; American Botanical Council: Austin, TX, USA, 1998.

89. Rietjens, I.M.; Boersma, M.G.; van der Woude, H.; Jeurissen, S.M.; Schutte, M.E.; Alink, G.M. Flavonoids and alkenylbenzenes: Mechanisms of mutagenic action and carcinogenic risk. Mutat. Res. 2005, 574, 124-138. [CrossRef]

90. Yancu, D.; Vaillancourt, C.; Sanderson, J.T. Evaluating the effects on steroidogenesis of estragole and trans-anethole in a feto-placental co-culture model. Mol. Cell. Endocrinol. 2019, 498, 110583. [CrossRef] [PubMed]

91. Yancu, D.; Sanderson, T. Essential oils disrupt steroidogenesis in a feto-placental co-culture model. Reprod. Toxicol. 2019, 90, 33-43. [CrossRef] [PubMed]

92. Nikolić, M.; Marković, T.; Mojović, M.; Pejin, B.; Savić, A.; Perić, T.; Marković, D.; Stević, T.; Soković, M. Chemical composition and biological activity of Gaultheria procumbens L. essential oil. Ind. Crop. Prod. 2013, 49, 561-567. [CrossRef]

93. Lapczynski, A.; Jones, L.; McGinty, D.; Bhatia, S.P.; Letizia, C.S.; Api, A.M. Fragrance material review on methyl salicylate. Food Chem. Toxicol. 2007, 45, 428-452. [CrossRef] [PubMed]

94. Heng, M.C. Local necrosis and interstitial nephritis due to topical methyl salicylate and menthol. Cutis 1987, 39, $442-444$.

95. Cross, S.E.; Anderson, C.; Thompson, M.J.; Roberts, M.S. Is there tissue penetration after application of topical salicylate formulations? Lancet 1997, 350, P636. [CrossRef]

96. Davison, C.; Zimmermann, E.F.; Smith, P.K. On the metabolism and toxicity of methyl salicylate. J. Pharmacol. Exp. Ther. 1961, 132, 207-211.

97. Ojiambo, H.P. Methylsalicylate myopathy in man. East Afr. Med. J. 1971, 48, 735-740. [PubMed]

98. Opdyke, D.L.J. Monographs on fragrance raw materials. Food Cosmet. Toxicol. 1978, 16, 56-59.

99. Adams, J.T.; Bigler, J.A.; Green, O.C. A case of methyl salicylate intoxication treated by exchange transfusion. J. Am. Med. Assoc. 1957, 165, 1563-1565. [CrossRef]

100. Zhang, Z.; Jia, C.; Hu, Y.; Sun, L.; Jiao, J.; Zhao, L.; Zhu, D.; Li, J.; Tian, Y.; Bai, H.; et al. The estrogenic potential of salicylate esters and their possible risks in foods and cosmetics. Toxicol. Lett. 2012, 209, 146-153. [CrossRef]

101. Ellenhorn, M.J.; Barceloux, D.G. Medical Toxicology: Diagnosis and Treatment of Human Poisoning; Elsevier Science: New York, NY, USA, 1988.

102. Wilson, J.G. Present status of drugs as teatogens in man. Teratology 1973, 7, 3-15. [CrossRef]

103. Karabulut, A.K.; Ulger, H.; Pratten, M. Protection by free oxygen radical scavenging enzymes against salicylate-induced embryonic malformations in vitro. Toxicol. In Vitro 2000, 14, 297-307. [CrossRef]

104. Kavlock, R.J.; Chernoff, N.; Rogers, E.; Whitehouse, D.; Carver, B.; Gray, J.; Robinson, K. An analysis of fetotoxicity using biochemical endpoints of organ differentiation. Teratology 1982, 26, 183-194. [CrossRef] [PubMed]

105. Woo, D.C.; Hoar, R.M. "Apparent hydronephrosis" as a normal aspect of renal development in late gestation of rats: The effect of methyl salicylate. Teratology 1972, 6, 191-196. [CrossRef]

106. Pyun, J.S. Effect of methyl salicylate on developing rat embryos. Ch'oesin Uihak 1970, 13, 63-72.

107. Daston, G.P.; Rehnberg, B.F.; Carver, B.; Rogers, E.H.; Kavlock, R.J. Functional Teratogens of the Rat Kidney: I. Colchicine, Dinoseb, and Methyl Salicylate. Toxicol. Sci. 1988, 11, 381-400. [CrossRef]

108. Warkany, J.; Takacs, E. Experimental Production of Congenital Malformations in Rats by Salicylate Poisoning. Am. J. Pathol. 1959, 35, 315-331. [PubMed]

109. Saito, H.; Yokoyama, A.; Takeno, S.; Sakai, T.; Ueno, K.; Masumura, H.; Kitagawa, H. Fetal Toxicity and Hypocalcemia Induced by Acetylsalicylic Acid Analogues. Res. Commun. Chem. Pathol. Pharmacol. 1982, 38, 209-220.

110. Overman, D.O.; White, J.A. Comparative Teratogenic Effects of Methyl Salicylate Applied Orally or Topically to Hamsters. Teratology 1983, 28, 421-426. [CrossRef] [PubMed]

111. Pages, N.; Fournier, G.; Baduel, C.; Tur, N.; Rusnac, M. Sabinyl Acetate, the Main Component of Juniperus sabina L'Hérit. Essential Oil, is Responsible for Antiimplantation Effect. Phyther. Res. 1996, 10, 438-440. [CrossRef]

112. Chamorro, G.; Salazar, M.; Fournier, G.; Pages, N. Anti-implantation effects of various extracts of Plectranthus fruticosus on pregnant rats. Planta Med. 1991, 57, 81. [CrossRef] [PubMed]

113. Pages, N.; Salazar, M.; Chamorro, G.; Fournier, G.; Paris, M.; Dumitresco, S.M.; Boudene, C. Teratological evaluation of Plectranthus fruticosus leaf essential oil. Planta Med. 1988, 54, 296-298. [CrossRef]

114. Pages, N.; Fournier, G.; Chamorro, G.; Salazar, M. Teratogenic effects of Plectranthus fruticosus essential oil in mice. Phyther. Res. 1991, 5, 94-96. [CrossRef]

115. Pages, N.; Fournier, G.; Velut, V.; Imbert, C. Potential teratogenicity in mice of the essential oil of Salvia lavandulifolia Vahl. Study of a fraction rich in sabinyl acetate. Phyther. Res. 1992, 6, 80-83. [CrossRef]

116. Pages, N.; Fournier, G.; Chamorro, G.; Salazar, M.; Paris, M.; Boudene, C. Teratological evaluation of Juniperus sabina essential oil in mice. Planta Med. 1989, 55, 144-146. [CrossRef] [PubMed] 
117. Grieve, M. A Modern Herbal; Penguin books: Harmondsworth, UK, 1978.

118. Chamorro, G.; Salazar, M.; Fournier, G.; Pages, N. The anti-implantation effects of various savine extracts on the pregnant rat. J. Toxicol. Clin. Exp. 1990, 10, 157-160.

119. Agrawal, O.P.; Bharadwaj, S.; Mathur, R. Antifertility Effects of Fruits of Juniperus Communis. Planta Med. 1980, 39, 98-101. [CrossRef] [PubMed]

120. Perry, N.S.; Houghton, P.J.; Sampson, J.; Theobald, A.E.; Hart, S.; Lis-Balchin, M.; Hoult, J.R.; Evans, P.; Jenner, P.; Milligan, S.; et al. In-vitro activity of S. lavandulaefolia (Spanish sage) relevant to treatment of Alzheimer's disease. J. Pharm. Pharmacol. 2001, 53, 1347-1356. [CrossRef]

121. Millet, Y.; Jouglard, J.; Steinmetz, M.D.; Tognetti, P.; Joanny, P.; Arditti, J. Toxicity of some essential plant oils. Clinical and experimental study. Clin. Toxicol. 1981, 18, 1485-1498. [CrossRef] [PubMed]

122. Küpeli Akkol, E.; Ilhan, M.; Ayşe Demirel, M.; Keleş, H.; Tümen, I.; Süntar, I. Thuja occidentalis L. and its active compound, $\alpha$-thujone: Promising effects in the treatment of polycystic ovary syndrome without inducing osteoporosis. J. Ethnopharmacol. 2015, 168, 25-30. [CrossRef] [PubMed]

123. Leung, A.Y.; Foster, S. Encyclopedia of Common Natural Ingredients Used in Food, Drugs and Cosmetics, 2nd ed.; John Wiley and Sons: Hoboken, NJ, USA, 2003.

124. Gerarde, H.W. Toxicology and Biochemistry of Aromatic Hydrocarbons; Elsevier: London, UK, 1960.

125. Budavari, S. The Merck Index, 11th ed.; Merck: Branchburg, NJ, USA, 1989.

126. Sampson, W.K.; Fernandez, G. Experimental convulsions in the rat. J. Pharm. Exp. Ther. 1939, 65, 275-280.

127. Hall, A.C.; Turcotte, C.M.; Betts, B.A.; Yeung, W.-Y.; Agyeman, A.S.; Burk, L.A. Modulation of human GABAA and glycine receptor currents by menthol and related monoterpenoids. Eur. J. Pharmacol. 2004, 506, 9-16. [CrossRef] [PubMed]

128. Szczot, M.; Czyzewska, M.M.; Appendino, G.; Mozrzymas, J.W. Modulation of GABAergic Synaptic Currents and Current Responses by $\alpha$-thujone and Dihydroumbellulone. J. Nat. Prod. 2012, 75, 622-629. [CrossRef]

129. Czyzewska, M.M.; Mozrzymas, J.W. Monoterpene $\alpha$-thujone exerts a differential inhibitory action on GABA A receptors implicated in phasic and tonic GABAergic inhibition. Eur. J. Pharmacol. 2013, 702, 38-43. [CrossRef]

130. Pelkonen, O.; Abass, K.; Wiesner, J. Thujone and thujone-containing herbal medicinal and botanical products: Toxicological assessment. Regul. Toxicol. Pharmacol. 2013, 65, 100-107. [CrossRef]

131. Margaria, R. Acute and Sub-acute Toxicity Study on Thujone. Unpublished report of the Istituto di Fisiologia, Università di Milano (cited from CoE Datasheet RD4.2/14-44, 1999); Istituto di Fisiologia, Università di Milano: Milano, Italy, 1963.

132. Abass, K.; Reponen, P.; Mattila, S.; Pelkonen, O. Metabolism of $\alpha$-thujone in Human Hepatic Preparations in Vitro. Xenobiotica 2011, 41, 101-111. [CrossRef]

133. Burkhard, P.R.; Burkhardt, K.; Haenggeli, C.A.; Landis, T. Plant-induced Seizures: Reappearance of an Old Problem. J. Neurol. 1999, 246, 667-670. [CrossRef] [PubMed]

134. Laadraoui, J.; Aboufatima, R.; El Gabbas, Z.; Ferehan, H.; Bezza, K.; Ait Laaradia, M.; Marhoume, F.; Wakrim, E.M.; Chait, A. Effect of Artemisia herba-alba consumption during pregnancy on fertility, morphological and behaviors of mice offspring. J. Ethnopharmacol. 2018, 226, 105-110. [CrossRef] [PubMed]

135. Almasad, M.M.; Qazan, W.S.; Daradka, H. Reproductive toxic effects of Artemisia herba alba ingestion in female Spague-Dawley rats. Pak. J. Biol. Sci. 2007, 10, 3158-3161. [PubMed]

136. Ciganda, C.; Laborde, A. Herbal infusions used for induced abortion. J. Toxicol. Clin. Toxicol. 2003, 41, 235-239. [CrossRef]

137. Lowenstein, L.; Ballew, D.H. Fatal acute haemolytic anaemia, thrombocytopenic purpura, nephrosis and hepatitis resulting from ingestion of a compound containing apiol. Can. Med. Assoc. J. 1958, 78, 195-198. [PubMed]

138. Laederich, L.; Mamou, H.; Arager, M. Fatal intoxication from apiol. Bull. Soc. Med. Hosp. Paris 1932, 48, 746-751.

139. Amerio, A.; De Benedictis, G.; Leondeff, J.; Mastrangelo, F.; Coratelli, P. Nephropathy due to apiol. Minerva Nefrol. 1968, 15, 49-70. (In Italian) [PubMed]

140. Patoir, A.; Patoir, G.; Bedrine, H. Le role abortif de l'apiol. Paris Med. 1936, 3, 442-446.

141. Riggs, J.; Hamilton, R.; Homel, S.; McCabe, J. Camphorated oil intoxication during pregnancy: Report of a case. Obs. Gynecol. 1965, 25, 255-258.

142. Phelan, W.J.I. Camphor poisoning: Over-the-counter dangers. Pediatrics 1976, 57, 428-431.

143. Banerjee, S.; Welsch, C.W.; Rao, A.R. Modulatory Influence of Camphor on the Activities of Hepatic Carcinogen Metabolizing Enzymes and the Levels of Hepatic and Extrahepatic Reduced Glutathione in Mice. Cancer Lett. 1995, 88, 163-169. [CrossRef]

144. Spector, W.S. Handbook of Toxicology. Vol 1. Acute Toxicities; WB Saunders: Philadelphia, PA, USA, 1956.

145. Siegel, E.; Wason, S. Camphor toxicity. Pediatr. Clin. N. Am. 1986, 33, 375-379. [CrossRef]

146. Smith, A.G.; Margolis, G. Camphor Poisoning; Anatomical and Pharmacologic Study; Report of a Fatal Case; Experimental Investigation of Protective Action of Barbiturate. Am. J. Pathol. 1954, 30, 857-869.

147. Merkulova, O.S. Reflex Mechanism of camphor and pyramidone experimental epilepsy. Dokl. Akad. Nauk. USSR 1957, 112, 968-971.

148. Wenzel, D.G.; Ross, C.R. Central Stimulating Properties of Some Terpenones. J. Am. Pharm. Assoc. 1957, 46, 77-82. [CrossRef]

149. Committee on Drugs Camphor revisited: Focus on toxicity (RE9422). Pediatrics 1994, 94, 127-128.

150. Rahimi, M.; Shokri, F.; Hassanian-Moghaddam, H.; Zamani, N.; Pajoumand, A.; Shadnia, S. Severe camphor poisoning, a seven-year observational study. Environ. Toxicol. Pharmacol. 2017, 52, 8-13. [CrossRef] 
151. Rabl, W.; Katzgraber, F.; Steinlechner, M. Camphor ingestion for abortion (case report). Forensic Sci. Int. 1997, 89, 137-140. [CrossRef]

152. Leuschner, J. Reproductive toxicity studies of d-camphor in rats and rabbits. Arzneimmittel-Forschung 1997, 47, 124-128.

153. Stojanović, N.M.; Randjelović, P.J.; Mladenović, M.Z.; Ilić, I.R.; Petrović, V.; Stojiljković, N.; Ilić, S.; Radulović, N.S. Toxic essential oils, part VI: Acute oral toxicity of lemon balm (Melissa officinalis L.) essential oil in BALB/c mice. Food Chem. Toxicol. 2019, 113, 110794. [CrossRef] [PubMed]

154. Opdyke, D.L.J. Citral. Food Chem. Toxicol. 1979, 17, 259-266.

155. Feron, V.J.; TiI, H.P.; de Vrijer, F.; Woutersen, R.A.; Cassee, F.R.; van Bladeren, P.J. Aldehydes: Occurrence, carcinogenic potential, mechanism of action and risk assessment. Mutat. Res. 1991, 259, 363-385. [CrossRef]

156. Toaff, M.E.; Abramovici, A.; Spom, J.; Liban, E. Selective oocyte degeneration and impaired fertility in rats treated with the ahphatic monoterpene, Citral. J. Reprod. Fertil. 1979, 55, 347-352. [CrossRef] [PubMed]

157. Kwon, H.-J.; Shin, J.-O.; Lee, J.-M.; Cho, K.-W.; Lee, M.-J.; Cho, S.-W.; Jung, H.-S. Retinoic acid modulates chondrogenesis in the developing mouse cranial base. J. Exp. Zool. B Mol. Dev. Evol. 2011, 316, 574-583. [CrossRef]

158. Koussoulakou, D.S.; Margaritis, L.H.; Koussoulakos, S.L. Antagonists of retinoic acid and BMP4 affect fetal mouse osteogenesis and odontoblast differentiation. Pathophysiology 2011, 18, 103-109. [CrossRef]

159. Tanaka, M.; Tamura, K.; Ide, H. Citral, an inhibitor of retinoic acid synthesis, modifies chick limb development. Dev. Biol. 1996, 175, 239-247. [CrossRef] [PubMed]

160. Di Renzo, F.; Broccia, M.L.; Giavini, E.; Menegola, E. Citral, an inhibitor of retinoic acid synthesis, attenuates the frequency and severity of branchial arch abnormalities induced by triazole-derivative fluconazole in rat embryos cultured in vitro. Reprod. Toxicol. 2007, 24, 326-332. [CrossRef]

161. Connor, M.J. Oxidation of retinol to retinoic acid as a requirement for biological activity in mouse epidermis. Cancer Res. 1988, 48 , 7038-7040. [PubMed]

162. Connor, M.J. Modulation of tumor promotion in mouse skin by the food additive citral (3,7-dimethyl-2,6-octadienal). Cancer Lett. 1991, 56, 25-28. [CrossRef]

163. Abramovici, A. Teratogenic effect of cosmetic eonstituents on the chick embryo. Adv. Exp. Med. Biol. 1972, 27, 161-174.

164. Abramovici, A.; Kam, J.; Liban, E.; Barishak, R.Y. Incipient histological lesions in citral-induced microphthalmos in chick embryos. Dev. Neurosci. 1978, 1, 177-185. [CrossRef]

165. Abramovici, A.; Rachmuth-Forschmidt, P.; Liban, E.; Andbank, U. Experimental limb dysmorphogenesis as a model of chemical injury response in undifferentiated embryonic tissues: A light and electron microscopical study. J. Pathol. 1980, 131, 289-308. [CrossRef]

166. Le Bouffant, R.; Guerquin, M.J.; Duquenne, C.; Frydman, N.; Coffigny, H.; Rouiller-Fabre, V.; Frydman, R.; Habert, R.; Livera, G. Meiosis initiation in the human ovary requires intrinsic retinoic acid synthesis. Hum. Reprod. 2010, 25, 2579-2590. [CrossRef] [PubMed]

167. Nogueira, A.C.M.A.; Carvalho, R.R.; Souza, C.A.M.; Chahoud, I.; Paumgartten, F.J. Study on the embryofeto-toxicity of citral in the rat. Toxicology 1995, 96, 105-113. [CrossRef]

168. Gaworski, C.L.; Vollmuth, T.A.; York, R.G.; Heck, J.D.; Aranyi, C. Developmental toxicity evaluation of inhaled citral in Sprague-Dawley rats. Food Chem. Toxicol. 1992, 30, 269-275. [CrossRef]

169. Griffith, M.; Zile, M.H. Retinoic acid, midkine, and defects of secondary neurulation. Teratology 2000, 62, 123-133. [CrossRef]

170. Schuh, T.J.; Hall, B.L.; Kraft, J.C.; Privalsky, M.L.; Kimelman, D. v-erbA and citral reduce the teratogenic effects of all-trans retinoic acid and retinol, respectively, in Xenopus embryogenesis. Development 1993, 119, 785-798. [PubMed]

171. Kronmiller, J.E.; Beeman, C.S.; Nguyen, T.; Berndt, W. Blockade of the initiation of murine odontogenesis in vitro by citral, an inhibitor of endogenous retinoic acid synthesis. Arch. Oral Biol. 1995, 40, 645-652. [CrossRef]

172. Geldof, A.A.; Engel, C.; Rao, B.R. Estrogenic action of commonly used fragrant agent citral induces prostatic hyperplasia. Urol. Res. 1992, 20, 139-144. [CrossRef]

173. Thomassen, D.; Knebel, N.; Slattery, J.T.; McClanahan, R.H.; Nelson, S.D. Reactive intermediates in the oxidation of menthofuran by cytochromes P-450. Chem. Res. Toxicol. 1992, 5, 123-130. [CrossRef] [PubMed]

174. Chen, W.; Lu, Y.; Gao, M.; Wu, J.; Wang, A.; Shi, R. Anti-angiogenesis effect of essential oil from Curcuma zedoaria in vitro and in vivo. J. Ethnopharmacol. 2011, 133, 220-226. [CrossRef] [PubMed]

175. Gordon, W.P.; Huitric, A.C.; Seth, C.L.; McClanahan, R.H.; Nelson, S.D. The metabolism of the abortifacient terpene, (R)-(+)pulegone, to a proximate toxin, menthofuran. Drug Metab. Dispos. 1987, 15, 589-594.

176. Chen, X.W.; Serag, E.S.; Sneed, K.B.; Zhou, S.F. Herbal bioactivation, molecular targets and the toxicity relevance. Chem. Biol. Interact. 2011, 192, 161-176. [CrossRef]

177. Cohen, S.M.; Eisenbrand, G.; Fukushima, S.; Gooderham, N.J.; Guengerich, F.P.; Hecht, S.S.; Rietjens, I.M.C.M.; Bastaki, M.; Davidsen, J.M.; Harman, C.L.; et al. FEMA GRAS assessment of natural flavor complexes: Mint, buchu, dill and caraway derived flavoring ingredients. Food Chem. Toxicol. 2020, 135, 110870. [CrossRef] [PubMed]

178. Moorthy, B.; Madyastha, P.; Madyastha, K.M. Hepatotoxicity of pulegone in rats: Its effects on microsomal enzymes, in vivo. Toxicology 1989, 55, 327-337. [CrossRef]

179. Gordon, W.P.; Forte, A.J.; McMurtry, R.J.; Gal, J.; Nelson, S.D. Hepatotoxicity and pulmonary toxicity of pennyroyal oil and its constituent terpenes in the mouse. Toxicol. Appl. Pharmacol. 1982, 65, 413-424. [CrossRef] 
180. Moorthy, B.; Vijayasarathi, S.K.; Basu, A.; Madyastha, K.M. Biochemical, histopathological and ultrastructural changes in rat liver induced by (R)-(+)-pulegone, a monoterpene ketone. Toxicol. Environ. Chem. 1991, 33, 121-131. [CrossRef]

181. Madyastha, P.; Moorthy, B. Pulegone-mediated hepatotoxicity: Evidence for covalent binding of (R)-(+)-14C-pulegone to microsomal proteins in vitro. Chem. Biol. Interact. 1989, 72, 325-333. [CrossRef]

182. Madyastha, P.; Moorthy, B.; Vaidyanathan, C.S.; Madyastha, K.M. In vivo and in vitro destruction of rat liver cytochrome P450 by a monoterpene ketone, pulegone. Biochem. Biophys. Res. Commun. 1985, 128, 921-927. [CrossRef]

183. Nair, B. Final report on the safety assessment of Mentha piperita (peppermint) oil, Mentha piperita (peppermint) leaf extract, Mentha piperita (peppermint) leaf, and Mentha piperita (peppermint) leaf water. Int. J. Toxicol. 2001, 20, 61-73. [PubMed]

184. Anderson, I.B.; Mullen, W.H.; Meeker, J.E.; Khojasteh-Bakht, S.C.; Oishi, S.; Nelson, S.D.; Blanc, P.D. Pennyroyal toxicity: Measurement of toxic metabolite levels in two cases and review of the literature. Ann. Intern. Med. 1996, 124, 726-734. [CrossRef] [PubMed]

185. Sullivan, J.B.J.; Rumack, B.H.; Thomas, H.J.; Peterson, R.G.; Bryson, P. Pennyroyal oil poisoning and hepatotoxicity. J. Am. Med. Assoc. 1979, 242, 2873-2874. [CrossRef]

186. Bradley, P.R. British Herbal Compendium; British Herbal Medicine Association: Bournemouth, UK, 1992.

187. Benezra, C.; Epstein, W.L. Molecular recognition patterns of sesquiterpene lactones in costus-sensitive patients. Contact Dermatitis 1986, 15, 223-230. [CrossRef] [PubMed]

188. Jeong, S.J.; Itokawa, T.; Shibuya, M.; Kuwano, M.; Ono, M.; Higuchi, R.; Miyamoto, T. Costunolide, a sesquiterpene lactone from Saussurea lappa, inhibits the VEGFR KDR/Flk-1 signaling pathway. Cancer Lett. 2002, 187, 129-133. [CrossRef]

189. Wang, C.Y.; Tsai, A.C.; Peng, C.Y.; Chang, Y.L.; Lee, K.H.; Teng, C.M.; Pan, S.L. Dehydrocostuslactone suppresses angiogenesis in vitro and in vivo through inhibition of Akt/GSK-3 $\beta$ and mTOR signaling pathways. PLoS ONE 2012, 7, e31195. [CrossRef] [PubMed]

190. Bamosa, A.O.; Ali, B.A.; Al-Hawsawi, Z.A. The effect of thymoquinone on blood lipids in rats. Indian J. Physiol. Pharmacol. 2002, 46, 195-201. [PubMed]

191. Yi, T.; Cho, S.G.; Yi, Z.; Pang, X.; Rodriguez, M.; Wang, Y.; Sethi, G.; Aggarwal, B.B.; Liu, M. Thymoquinone inhibits tumor angiogenesis and tumor growth through suppressing AKT and extracellular signal-regulated kinase signaling pathways. Mol. Cancer Ther. 2008, 7, 1789-1796. [CrossRef] [PubMed]

192. AbuKhader, M.M. Thymoquinone in the clinical treatment of cancer: Fact or fiction? Pharmacogn. Rev. 2013, 7, 117-120. [CrossRef] [PubMed]

193. Al-Enazi, M.M. Effect of thymoquinone on malformations and oxidative stress-induced diabetic mice. Pak. J. Biol. Sci. 2007, 10, 3115-3119.

194. Chen, W.; Lu, Y.; Wu, J.; Gao, M.; Wang, A.; Xu, B. Beta-elemene inhibits melanoma growth and metastasis via suppressing vascular endothelial growth factor-mediated angiogenesis. Cancer Chemother. Pharmacol. 2011, 67, 799-808. [CrossRef]

195. Liu, J.; Zhang, Z.; Gao, J.; Xie, J.; Yang, L.; Hu, S. Downregulation effects of betaelemene on the levels of plasma endotoxin, serum TNF-alpha, and hepatic CD14 expression in rats with liver fibrosis. Front. Med. 2011, 5, 101-105. [CrossRef] [PubMed]

196. Yan, B.; Zhou, Y.; Feng, S.; Lv, C.; Xiu, L.; Zhang, Y.; Shi, J.; Li, Y.; Wei, P.; Qin, Z. $\beta$-Elemene-Attenuated Tumor Angiogenesis by Targeting Notch-1 in Gastric Cancer Stem-Like Cells. Evid. Based Complement. Altern. Med. 2013, 2013, 268468. [CrossRef]

197. Li, Q.Q.; Wang, G.; Huang, F.; Banda, M.; Reed, E. Antineoplastic effect of beta-elemene on prostate cancer cells and other types of solid tumour cells. J. Pharm. Pharmacol. 2010, 62, 1018-1027. [CrossRef] [PubMed]

198. Yang, H.; Wang, X.P.; Yu, L.L.; Zheng, S. The antitumor activity of elemene is associated with apoptosis. Zhonghua Zhongliu Zazhi 1996, 18, 169-172. [CrossRef] [PubMed]

199. Tan, P.; Zhong, W.; Cai, W. Clinical study on treatment of 40 cases of malignant brain tumor by elemene emulsion injection. Zhongguo Zhong Xi Yi Jie He Za Zhi 2000, 20, 645-648.

200. Zhong, Z.F.; Hoi, P.M.; Wu, G.S.; Xu, Z.T.; Tan, W.; Chen, X.P.; Cui, L.; Wu, T.; Wang, Y.T. Anti-angiogenic effect of furanodiene on HUVECs in vitro and on zebrafish in vivo. J. Ethnopharmacol. 2012, 141, 721-727. [CrossRef]

201. Tsuneki, H.; Ma, E.-L.; Kobayashi, S.; Sekizaki, N.; Maekawa, K.; Sasaoka, T.; Wang, M.-W.; Kimura, I. Antiangiogenic activity of beta-eudesmol in vitro and in vivo. Eur. J. Pharmacol. 2005, 512, 105-115. [CrossRef] [PubMed]

202. Domaracký, M.; Rehák, P.; Juhás, S.; Koppel, J. Effects of selected plant essential oils on the growth and development of mouse preimplantation embryos in vivo. Physiol. Res. 2007, 56, 97-104.

203. Dante, G.; Facchinetti, F. Herbal treatments for alleviating premenstrual symptoms: A systematic review. J. Psychosom. Obs. Gynecol. 2011, 32, 42-51. [CrossRef] [PubMed]

204. Chopin Lucks, B. Vitex agnus-castus essential oil and menopausal balance: A self-care survey. Complement. Ther. Nurs. Midwifery 2002, 8, 148-154. [CrossRef] [PubMed]

205. Chopin Lucks, B. Vitex agnus castus essential oil and menopausal balance: A research update. Complement Ther Nurs Midwifery 2003, 9, 157-160. [CrossRef]

206. van Die, M.D.; Burger, H.G.; Teede, H.J.; Bone, K. Vitex agnus-castus (Chaste-Tree = Berry) in the Treatment of Menopause-Related Complaints. J. Alt. Compl. Med. 2009, 15, 853-862. [CrossRef] [PubMed]

207. O'Mullane, N.M.; Joyce, P.; Kamath, S.V.; Tham, M.K.; Knass, D. Adverse CNS effects of menthol-containing olbas oil. Lancet $1982,1,1121$. 
208. Höld, K.M.; Sirisoma, N.S.; Sparks, S.E.; Casida, J.E. Metabolism and mode of action of cis- and trans-3-pinanones (the active ingredients of hyssop oil). Xenobiotica 2002, 32, 251-265. [CrossRef]

209. Sarici, S.U.; Kul, M.; Candemir, G.; Aydin, H.İ.; Alpay, F.; Gökçay, E. Neonatal convulsion after accidental ingestion of sage oil: A case report. Gulhane Med. J. 2004, 46, 161-162.

210. Halicioglu, O.; Astarcioglu, G.; Yaprak, I.; Aydinlioglu, H. Toxicity of Salvia officinalis in a newborn and a child: An alarming report. Pediatr Neurol. 2011, 45, 259-260. [CrossRef] [PubMed]

211. Ema, M.; Harazono, A.; Fujii, S.; Kawashima, K. Evaluation of developmental toxicity of $\beta$-thujaplicin (hinokitiol) following oral administration during organogenesis in rats. Food Chem. Toxicol. 2004, 42, 465-470. [CrossRef]

212. Dong, J.Y.; Xue, L.Q.; Zhu, X.W.; Zhou, Y. Antifertility agents from seeds of Daucus carota. Zhongcaoyao 1981, $12,61$.

213. Chu, Y.H.; Zhou, M.H.; Li, Q.; Bao, Y.M. Antifertility effect of volatile oil of Daucus carota seeds. Reprod. Contracept. 1985, 5, 37-40.

214. Zhou, N.N.; Mao, X.J.; Zhang, J.; Yang, S.W.; Zhang, J.M. Pharmacological investigation on contraindication of Curcuma zedoaria. Chin. Arch. Tradit. Chin. Med. 2004, 22, 2291-2294.

215. Kong, Y.C.; Xie, J.X.; But, P.P.H. Fertility regulating agents from traditional Chinese medicine. J. Ethnopharmacol. 1986, 15, 1-44. [CrossRef]

216. Farnsworth, N.R.; Bingel, A.S.; Cordell, G.A.; Crane, F.A.; Fong, H.H. Potential Value of Plants as Sources of New Antifertility Agents I. J. Pharm. Sci. 1975, 64, 535-598. [CrossRef] [PubMed] 